\title{
Controller Parameterization for Disturbance Response Decoupling: Application to Vehicle Active Suspension Control
}

\author{
Malcolm C. Smith, Fellow, IEEE, and Fu-Cheng Wang, Student Member, IEEE
}

\begin{abstract}
This paper derives a parameterization of the set of all stabilizing controllers for a given plant which leaves some prespecified closed-loop transfer function fixed. This result is motivated by the need to independently shape several different disturbance transmission paths in vehicle active suspension control. The result is studied in the context of quarter-, half-, and full-car vehicle models, to derive appropriate controller structures. A controller design is carried out for the full-car case and simulated with a nonlinear vehicle dynamics model.
\end{abstract}

Index Terms-Active suspension, controller parameterization, disturbance response decoupling, $H_{\infty}$, loop-shaping, vehicle dynamics.

\section{INTRODUCTION}

$\mathbf{T}$ HIS paper studies the problem of control system design when there are independent performance goals for several disturbance transmission paths. We are motivated by the problem of vehicle active suspension control where a key feature is the need to insulate the vehicle body from both road irregularities and load disturbances (e.g., inertial loads induced by braking and cornering). It is well known that these are conflicting requirements when passive suspensions are used, but the conflict may be removed when active control is employed with appropriate hardware structure, e.g., choice of sensor location, number, and type. Once a suitable hardware structure is selected, there remains the problem of designing the controller to achieve satisfactory responses for all of the disturbance transmission paths. It is this latter problem that is the main subject of this paper. To this end, we consider the problem of parameterizing the set of all stabilizing controllers for a given plant which leaves the transfer function for a given disturbance transmission path the same as when some nominal stabilizing controller is employed. In this way, the design for each disturbance path can be carried out successively, providing there is sufficient freedom to adjust the responses independently.

\section{A. Vehicle Active Suspension Control}

The use of active suspension on road vehicles has been considered for many years [8], [15], [17], [20], [26]. A large

Manuscript received November 30, 2000; revised July 31, 2001. Manuscript received in final form October 23, 2001. Recommended by Associate Editor M. Jankovic.

The authors are with the Department of Engineering, University of Cambridge, Cambridge CB2 1PZ, U.K. (e-mail: mcs@eng.cam.ac.uk; fcw22@eng.cam.ac.uk).

Publisher Item Identifier S 1063-6536(02)01768-2. number of different arrangements from semiactive to fully active schemes has been investigated [2], [16], [25], [27]. There has also been interest in characterizing the degrees of freedom and constraints involved in active suspension design. Constraints on the achievable response have been investigated from "invariant point," transfer-function, and energy/passivity points of view in [7], [9], [10], [18], [19]. In [18], a complete set of constraints was derived on the road and load disturbance response transfer-functions and results on the choice of sensors needed to achieve these degrees of freedom independently were obtained for the quarter-car model (see [4] for generalization of these results to half- and full-car models). In [19] it was shown that the road and load disturbance responses could not be adjusted independently for any passive suspension applied to a quarter-car model.

The need to design the road and load disturbance responses independently has been considered elsewhere in the active suspension literature. For example, in [15] a hardware and sensing arrangement was devised so that the feedback part of the scheme would not affect the response to road disturbances, which were designed to be suitably soft by means of passive elements in the scheme. In [24], [25] the actuator was placed in series with a spring and damper, which were chosen to give a suitably soft response to road irregularities in the absence of a feedback signal. A controller structure using a filtered combination of the sensor measurements was then selected so that the road disturbance responses were unaffected by the feedback. The present paper represents a continuation of this idea by finding in general the required controller structure to achieve this property for any set of measurements.

In active suspension design for full-car models, it has been found advantageous to decompose the motion into bounce, pitch and roll components for the vehicle body and additionally warp for the wheels in contact with the road [6], [12], [13], [19]. This paper will also exploit such transformations, at least to a partial extent. In the full-car case we will exploit symmetry to decompose into the bounce/pitch and roll/warp half-cars. In the half-car case we will use our results to determine the feedback structure to allow road and load disturbances to be shaped independently and discuss the simplicity assumptions which allow a further decomposition of the half-car into two quarter-cars.

\section{B. Controller Parameterization}

The idea of parameterizing all stabilizing controllers in a linear feedback system is a standard one [28], [30]. The extension of this idea to two-degree-of-freedom schemes 
(which allows the response to reference commands and the return ratio of the feedback path to be optimized independently) is also standard [21], [29]. The generalization to additional degrees-of-freedom to include some exogenous disturbances has also been considered [14]. The parameterization of all stabilizing controllers which leaves some prespecified closed-loop transfer function fixed, as considered in this paper, represents a continuation of these ideas and techniques.

Our approach makes use of algebraic properties of the ring of stable, proper rational functions [21]. To facilitate the parameterization at the required level of generality we will introduce the idea of left and right normal rank factorizations of a rational matrix (Definition 1).

\section{Outline of Paper}

Section II sets up in generality the problem of parameterizing all stabilizing controllers which leave some prespecified closed-loop transfer function fixed. Our basic results, which characterize the required structure of the Youla $Q$-parameter, are given in Theorems 1 and 2. Section III considers the standard quarter-car model employing a "Sharp" actuator with various choices of measured variables. The required controller structures to leave the road disturbance responses the same as in the passive case are derived using the results of Section II. Section IV considers a simple half-car model with acceleration and strut deflection measurements, and again derives the control structure required to keep the road disturbance responses the same as in the passive case. "Simplicity" conditions which allow the design to be carried out for two separate quarter-cars are presented. Section V considers a simple linearized full-car model and shows how this may be separated into two half-car models under a mild symmetry assumption. Section VI presents a design for the full-car model with acceleration and strut deflection measurements. The bounce/pitch half-car is treated according to the theory in Section IV. The roll and warp modes are each treated as quarter-cars with the warp mode being handled in a special way. The controller design is simulated with a nonlinear vehicle model using the multibody simulation package AutoSim.

\section{Controller Parametrisation Results}

We consider the LFT (linear fractional transformation) model in Fig. 1, where the Laplace transfer function of the generalized plant $P$ is partitioned as

$$
P=\left[\begin{array}{ll}
P_{11} & P_{12} \\
P_{21} & P_{22}
\end{array}\right]
$$

and further partitioned conformably with the disturbance signals as

$$
\left.\left[\begin{array}{c}
\hat{z}_{1} \\
\hat{z}_{2} \\
\hat{y}
\end{array}\right]=\left[\begin{array}{cc}
{\left[\begin{array}{ll}
P_{11,11} & P_{11,12} \\
P_{11,21} & P_{11,22}
\end{array}\right]} & {\left[\begin{array}{c}
P_{12,1} \\
P_{12,2}
\end{array}\right]} \\
P_{21,1} & P_{21,2}
\end{array}\right] \quad\left[\begin{array}{c}
\hat{w}_{1} \\
\hat{w}_{22}
\end{array}\right]\right]
$$

where $w_{1} \in \mathbb{R}^{m_{1}}, w_{2} \in \mathbb{R}^{m_{2}}, u \in \mathbb{R}^{m_{3}}, z_{1} \in \mathbb{R}^{p_{1}}, z_{2} \in \mathbb{R}^{p_{2}}$, $y \in \mathbb{R}^{p_{3}}$ at any time instant $\hat{u}$ denotes the Laplace transform of $u(t)$ etc. We consider the problem of parameterizing all stabilizing controllers which leave $T_{\hat{w}_{1} \rightarrow \hat{z}_{1}}$ (the transfer function from $\hat{w}_{1}$ to $\hat{z}_{1}$ ) the same as for some given stabilizing controller $K_{0}$.

Let $P_{22}=N M^{-1}=\tilde{M}^{-1} \tilde{N}$ be the right and left coprime factorizations of $P_{22}$ over $\mathbb{R} \mathbb{H}_{\infty}$. Then all stabilizing controllers can be parameterized by

$$
\begin{aligned}
K & =(Y-M Q)(X-N Q)^{-1} \\
& =(\tilde{X}-Q \tilde{N})^{-1}(\tilde{Y}-Q \tilde{M})
\end{aligned}
$$

for $Q \in \mathbb{R} \|_{\infty}^{m_{3} \times p_{3}}$ where $X, Y, \tilde{X}, \tilde{Y}$ are matrices with elements in $\mathbb{R} H_{\infty}$ which satisfy the Bezout identity

$$
\left[\begin{array}{cc}
\tilde{X} & -\tilde{Y} \\
-\tilde{N} & \tilde{M}
\end{array}\right]\left[\begin{array}{ll}
M & Y \\
N & X
\end{array}\right]=I
$$

For our first result (Theorem 1) it is convenient if the factorizations are chosen so that $Q=0$ corresponds to the desired stabilizing controller, i.e., $K_{0}=Y X^{-1}=\tilde{X}^{-1} \tilde{Y}$. (This assumption will be relaxed in the corollary to Theorem 2.)

The results we will establish in this section make use of certain algebraic properties of the set $\mathbb{R} \mathbb{H}_{\infty}$, namely its ring structure. The reader is referred to [21] for the necessary background on this topic. Here we will be content to recall a few facts. The set $\mathbb{R} \mathbb{H}_{\infty}$ has the property of being a Euclidean domain with degree function defined by the total number of zeros of the element in the closed right half plane and at infinity (counting multiplicities). The invertible elements in $\mathbb{R} H_{\infty}$ are called units, and are the elements with degree equal to zero. A matrix $U \in \mathbb{R} \mathbb{R}_{\infty}^{m \times m}$ is called unimodular if it has an inverse whose elements belong to $\mathbb{R} \mathbb{H}_{\infty}$, or equivalently, if its determinant is a unit in $\mathbb{R} \mathbb{H}_{\infty}$. The normal rank of a matrix $Q \in \mathbb{R} \mathbb{H}_{\infty}^{m \times n}$, denoted normal $\operatorname{rank}(Q(s))$, is the maximum rank of $Q(s)$ for any $s \in \mathbb{C}$ which is not a pole. Equivalently, the normal rank is equal to the rank for almost all $s \in \mathbb{C}$. We now introduce a type of matrix factorization which will be useful in proving the subsequent results.

Definition 1: Let $T$ be a matrix with elements in $\mathbb{R} \mathbb{H}_{\infty} . T$ is said to have a left normal rank factorization (lnf) if there exist matrices $E$ and $V=\left(V_{1}^{\prime}, V_{2}^{\prime}\right)^{\prime}$ over $\mathbb{R R} \mathbb{H}_{\infty}$ with $E$ of full column normal rank and $V$ unimodular such that $T=E V_{1} . T$ is said to have a right normal rank factorization (rnf) if there exist matrices $F$ and $U=\left(U_{1}, U_{2}\right)$ over $\mathbb{R} \mathbb{H}_{\infty}$ with $F$ of full row normal rank and $U$ unimodular such that $T=U_{1} F$.

Lemma 1: For any $T \in \mathbb{R} \mathbb{R}_{\infty}^{r \times c}$, there exists an lnf and an $\mathrm{rnf}$ of $T$.

Proof: See the Appendix.

We return to the problem of parameterizing all stabilizing controllers which leave the transfer function $T_{\hat{w}_{1} \rightarrow \hat{z}_{1}}$ the same as when the controller $K_{0}=Y X^{-1}=\tilde{X}^{-1} \tilde{Y}$ is applied. From [5], the closed-loop transfer function in Fig. 1 can be expressed as

$$
T\left(\begin{array}{l}
\hat{w}_{1} \\
\hat{w}_{2}
\end{array}\right) \rightarrow\left(\begin{array}{l}
\hat{z}_{1} \\
\hat{z}_{2}
\end{array}\right)=T_{1}-T_{2} Q T_{3}
$$

where $T_{1}, T_{2}, T_{3}$ have elements in $\mathbb{R} H_{\infty}$ and are given by

$$
\begin{aligned}
& T_{1}=P_{11}+P_{12} M \tilde{Y} P_{21} \\
& T_{2}=P_{12} M \\
& T_{3}=\tilde{M} P_{21} .
\end{aligned}
$$




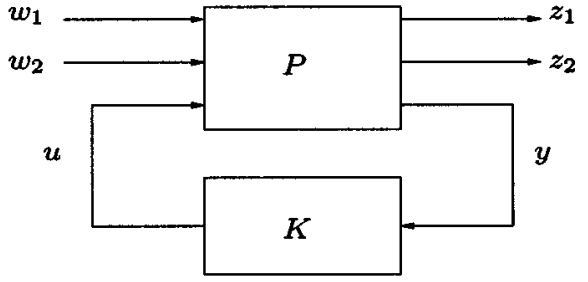

Fig. 1. Generalized model in LFT form.

Thus the problem reduces to parameterizing all stabilizing controllers which leave $T_{\hat{w}_{1} \rightarrow \hat{z}_{1}}=\left(T_{1}\right)_{11}$. These are characterized by all $Q \in \mathbb{R} \mathbb{R} \prod_{\infty}^{m_{3} \times p_{3}}$ such that $\left(T_{2} Q T_{3}\right)_{11}=T_{2,1} Q T_{3,1}=0$, where $T_{2,1}=P_{12,1} M$ and $T_{3,1}=\tilde{M} P_{21,1}$. We now introduce an $\operatorname{lnf}$ of $T_{2,1}$ and an $\operatorname{rnf}$ of $T_{3,1}$ as follows:

$$
\begin{aligned}
& T_{2,1}:=E V_{1} \\
& T_{3,1}:=U_{1} F
\end{aligned}
$$

where $E \in \mathbb{R} \mathbb{R}_{\infty}^{p_{1} \times r_{2}}, F \in \mathbb{R} \mathbb{R}_{\infty}^{r_{3} \times m_{1}}, U_{1} \in \mathbb{R} \mathbb{R}_{\infty}^{p_{3} \times r_{3}}, V_{1} \in$ $\mathbb{R} \mathbb{R} \mathbb{H}_{\infty}^{r_{2} \times m_{3}}, r_{2}$ and $r_{3}$ are the normal rank of $T_{2,1}$ and $T_{3,1}$ respectively. Note that $r_{2}$ and $r_{3}$ are also the normal rank of $P_{12,1}$ and $P_{21,1}$, respectively. Furthermore, we have the inequalities $r_{2} \leq \min \left(p_{1}, m_{3}\right), r_{3} \leq \min \left(m_{1}, p_{3}\right)$.

Theorem 1: Consider any stabilizable $P$ in the configuration of Fig. 1. All stabilizing controllers such that the closed-loop transfer function $T_{\hat{w}_{1} \rightarrow \hat{z}_{1}}=\left(T_{1}\right)_{11}$ are given by $K$ expressed in the form of (2) and (3) with

$$
Q=\left(\begin{array}{c}
V_{1} \\
V_{2}
\end{array}\right)^{-1}\left(\begin{array}{c}
Q_{1} \widetilde{U}_{2} \\
Q_{2}
\end{array}\right)
$$

for $Q_{1} \in \mathbb{R} \mathbb{H}_{\infty}^{r_{2} \times\left(p_{3}-r_{3}\right)}$ and $Q_{2} \in \mathbb{R} \mathbb{H}_{\infty}^{\left(m_{3}-r_{2}\right) \times p_{3}}, V_{1}$ and $U_{1}$ defined from the $\operatorname{lnf}$ and $\mathrm{rnf}$ factorizations (5) and (6), $U_{2}, V_{2}$ are chosen such that $\left(U_{1}, U_{2}\right)$ and $\left(V_{1}^{\prime}, V_{2}^{\prime}\right)^{\prime}$ are unimodular, and $\widetilde{U}_{2} \in \mathbb{R R} \prod_{\infty}^{\left(p_{3}-r_{3}\right) \times p_{3}}$ is a partition of $U^{-1}$

$$
U^{-1}=\left(U_{1}, U_{2}\right)^{-1}=\left(\begin{array}{c}
\widetilde{U}_{1} \\
\widetilde{U}_{2}
\end{array}\right)
$$

Proof: See the Appendix.

The control structure given in (7) is arrived at by completing the matrix $U_{1}$ to a unimodular matrix and then extracting $\widetilde{U}_{2}$ from the resulting matrix inverse. Since $U_{1}$ and the completion are not unique then neither is $\widetilde{U}_{2}$. It will be useful to characterize this nonuniqueness in terms of the parameterization of the set $\left\{Q_{1} \widetilde{U}_{2}: Q_{1} \in \mathbb{R} \mathbb{R} \mathbb{r}_{\infty}^{r_{2} \times\left(p_{3}-r_{3}\right)}\right\}$ directly. This is done in the following lemma.

Lemma 2: Given two sets $A=\left\{Q_{1} \widetilde{U}_{2}: Q_{1} \in \mathbb{R} \mathbb{H}_{\infty}^{r \times c}\right\}$ and $B=\left\{Q_{1}^{\dagger} \widetilde{U}_{2}^{\dagger}: Q_{1}^{\dagger} \in \mathbb{R} \mathbb{H}_{\infty}^{r \times c}\right\}$ where $\widetilde{U}_{2}, \widetilde{U}_{2}^{\dagger} \in \mathbb{R} \mathbb{H}_{\infty}^{c \times q}$ are full row normal rank, then

1) $B \subseteq A$ if and only if $\widetilde{U}_{2}^{\dagger}$ is a left multiple of $\widetilde{U}_{2}$ over $\mathbb{R} \mathbb{W}_{\infty}$, i.e., there exists a $W \in \mathbb{R} \mathbb{H}_{\infty}^{c \times c}$ such that $\widetilde{U}_{2}^{\dagger}=$ $W \widetilde{U}_{2}$.

2) $B=A$ if and only if there exists a unimodular matrix $W \in \mathbb{R} \mathbb{H}_{\infty}^{c \times c}$ such that $\widetilde{U}_{2}^{\dagger}=W \widetilde{U}_{2}$.

Proof: See the Appendix.

Throughout this paper the vehicle dynamics examples will satisfy some special assumptions on the open-loop plant. This allows the controller parameterization of Theorem 1 to take a simplified form, and it turns out that a further useful structural simplification can then be made. It will be convenient to summarize these simplifications in the theorem below, which will then be applied directly throughout the paper. (The first two special assumptions on the open-loop plant arise because of some passive elements in the suspension system which ensure that the road disturbance responses are satisfactory without any feedback control. The third assumption is a rather technical one which says that the number of outputs to be left invariant is no smaller than the number of actuators and that this transmission path has full normal rank.)

Theorem 2: Let (i) $P$ be (open-loop) stable, (ii) $K_{0}=0$, (iii) $m_{3}=r_{2}$.

1) There exists $\widetilde{U}_{2} \in \mathbb{R} \mathbb{R}_{\infty}^{\left(p_{3}-r_{3}\right) \times p_{3}}$ such that all stabilizing controllers which give $T_{\hat{w}_{1} \rightarrow \hat{z}_{1}}=\left(T_{1}\right)_{1,1}$ can be parameterized as

$$
K=-\left(I-Q_{1} \widetilde{U}_{2} P_{22}\right)^{-1} Q_{1} \widetilde{U}_{2}
$$

for $Q_{1} \in \mathbb{R} \mathbb{H}_{\infty}^{r_{2} \times\left(p_{3}-r_{3}\right)}$.

2) A particular $\widetilde{U}_{2} \in \mathbb{R} \mathbb{H}_{\infty}^{\left(p_{3}-r_{3}\right) \times p_{3}}$ for which (8) parameterizes all stabilizing controllers such that $T_{\hat{w}_{1} \rightarrow \hat{z}_{1}}=$ $\left(T_{1}\right)_{1,1}$ can be calculated as follows: choose $M=I$, $\tilde{M}=I, N=\tilde{N}=P_{22}, X=I, \tilde{X}=I, Y=0$, $\tilde{Y}=0$ and $V_{1}=I$, define $U_{1}$ from the $\operatorname{rnf}(6)$, and calculate $U_{2}, \widetilde{U}_{2}$ as in Theorem 1 .

3) Consider any $\widetilde{U}_{2}^{\dagger}$ such that $K^{\dagger}=-\left(I-Q_{1} \widetilde{U}_{2}^{\dagger} P_{22}\right)^{-1}$ $Q_{1} \widetilde{U}_{2}^{\dagger}$ parameterizes all stabilizing controllers which give $T_{\hat{w}_{1} \rightarrow \hat{z}_{1}}=\left(T_{1}\right)_{1,1}$. Then there exist a unimodular matrix $W$ such that $\widetilde{U}_{2}^{\dagger}=W \widetilde{U}_{2}$, where $\widetilde{U}_{2}$ is defined in 2).

4) Let $\widetilde{U}_{2}$ be defined in 2) and let $K_{1}$ be any stabilizing controller for $\widetilde{U}_{2} P_{22}$. Then $K_{1} \widetilde{U}_{2}$ is a stabilizing controller for $P_{22}$ for which $T_{\hat{w}_{1} \rightarrow \hat{z}_{1}}=\left(T_{1}\right)_{1,1}$.

5) Let $\widetilde{U}_{2}$ be defined in 2 ) and consider any stabilizing controller $K$ for $P_{22}$ for which $T_{\hat{w}_{1} \rightarrow \hat{z}_{1}}=\left(T_{1}\right)_{1,1}$. Then we can write $K=K_{1} \widetilde{U}_{2}$, where $K_{1}$ is a stabilizing controller for $\widetilde{U}_{2} P_{22}$.

Proof: See the Appendix.

Corollary 1: Let conditions (i) and (iii) of Theorem 2 hold and suppose that $K_{0}=-\left(I-\bar{Q} P_{22}\right)^{-1} \bar{Q}$ for some $\bar{Q} \in \mathbb{R} \mathbb{H}_{\infty}^{m_{3} \times p_{3}}$. Then all stabilizing controllers which leave $(T)_{\hat{w}_{1} \rightarrow \hat{z}_{1}}$ the same as when $K_{0}$ is applied can be parameterized as

$$
K=-\left(I-\left(\bar{Q}+Q_{1} \widetilde{U}_{2}\right) P_{22}\right)^{-1}\left(\bar{Q}+Q_{1} \widetilde{U}_{2}\right)
$$

for some $Q_{1} \in \mathbb{R} \mathbb{R}_{\infty}^{r_{2} \times\left(p_{3}-r_{3}\right)}$ and $\widetilde{U}_{2}$ defined in Theorem 2 (2).

Proof: See the Appendix.

The controller structure given in (8), which is a special case of the general parameterization given in Theorem 1, may be represented in the block diagram form shown in Fig. 2(a). Theorem $2(4,5)$ shows that the essential feature in this controller structure is the presence of $\widetilde{U}_{2}$ as the rightmost term in (8). This is illustrated in the block diagram Fig. 2(b) where $K_{1}$ may be any stabilizing controller for the transformed plant $P^{\dagger}$. 


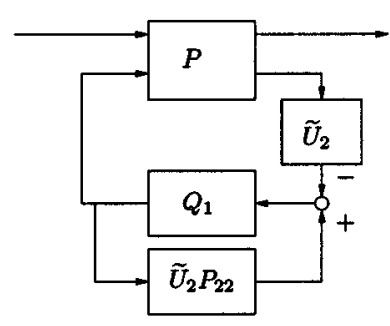

(a)

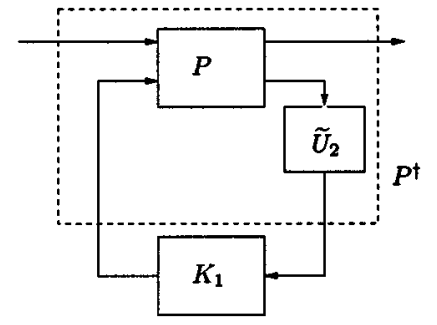

(b)
Fig. 2. (a) General controller structure. (b) Equivalent controller.

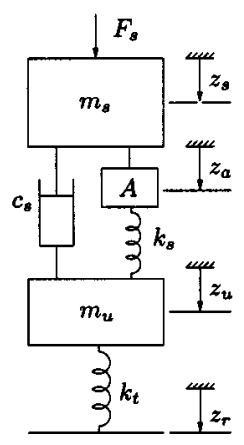

Fig. 3. The quarter-car model.

\section{THE QUARTER-CAR MODEL}

\section{A. The Quarter-Car With Two Measurements}

We begin with the quarter-car model of Fig. 3 where the sprung and unsprung masses are $m_{s}$ and $m_{u}$ and the tire is modeled as a linear spring with constant $k_{t}$. The suspension consists of a passive damper of constant $c_{s}$ in parallel with a series combination of an actuator $A$ and a spring of constant $k_{s}$ (sometimes referred to as a "Sharp" actuator [27]). Following [16] the actuator is modeled so that the relative displacement across the actuator will be a low-pass filtered version of the actuator's command signal, i.e.,

$$
\hat{z}_{s}-\hat{z}_{a}=\gamma(s) \hat{u} .
$$

As in [16], we use a second-order filter to represent the actuator dynamics

$$
\gamma(s)=\frac{\omega_{n}^{2}}{s^{2}+2 \delta \omega_{n} s+\omega_{n}^{2}} .
$$

The external disturbances are taken to be a load $F_{s}$ and a road displacement $z_{r}$, and the measurements are taken to be $\ddot{z}_{s}$ and $z_{s}-z_{u}$. The dynamic equations of the model are given by

$$
\begin{aligned}
m_{s} \ddot{z}_{s} & =F_{s}-u_{p} \\
m_{u} \ddot{z}_{u} & =u_{p}+F_{r}
\end{aligned}
$$

where

$$
\begin{aligned}
& u_{p}=c_{s}\left(\dot{z}_{s}-\dot{z}_{u}\right)+k_{s}\left(z_{a}-z_{u}\right) \\
& F_{r}=k_{t}\left(z_{r}-z_{u}\right) .
\end{aligned}
$$

We wish to parameterize all controllers which leave the transmission path from the road disturbance to $z_{s}$ and $z_{u}$ the same as in the open-loop, i.e., with $u=0$. This assumes that $k_{s}$ and $c_{s}$ are chosen to give satisfactory responses for this transmission path. In effect, this gives the choice of $K_{0}=0$. We now write the system in the form of Fig. 1 with $z_{1}=\left[z_{s}, z_{u}\right]^{\prime}, w_{1}=z_{r}$, $w_{2}=F_{s}, y=\left[\ddot{z}_{s}, z_{s}-z_{u}\right]^{\prime}, u$ equals the actuator command signal as in (10) and $z_{2}$ omitted. The corresponding dimensions are $m_{1}=m_{2}=m_{3}=1, p_{1}=2, p_{2}=0, p_{3}=2$. Equation (1) then takes the form

$$
\left[\begin{array}{c}
\hat{z}_{s} \\
\hat{z}_{u} \\
\hline s^{2} \hat{z}_{s} \\
\hat{z}_{s}-\hat{z}_{u}
\end{array}\right]=\left[\begin{array}{ll}
P_{11} & P_{12} \\
P_{21} & P_{22}
\end{array}\right]\left[\begin{array}{c}
\hat{z}_{r} \\
\hat{F}_{s} \\
\hat{\hat{u}}
\end{array}\right]
$$

with the transfer functions being given by

$$
\begin{aligned}
& P_{11}=\frac{1}{d(s)}\left[\begin{array}{cc}
k_{t}\left(c_{s} s+k_{s}\right) & p_{1}(s) \\
k_{t}\left(m_{s} s^{2}+c_{s} s+k_{s}\right) & \left(c_{s} s+k_{s}\right)
\end{array}\right] \\
& P_{12}=\frac{\gamma(s)}{d(s)}\left[\begin{array}{c}
k_{s}\left(m_{u} s^{2}+k_{t}\right) \\
-k_{s} m_{s} s^{2}
\end{array}\right] \\
& P_{21}=\frac{1}{d(s)}\left[\begin{array}{cc}
k_{t}\left(c_{s} s+k_{s}\right) s^{2} & p_{1}(s) s^{2} \\
-k_{t}\left(m_{s} s^{2}\right) & \left(m_{u} s^{2}+k_{t}\right)
\end{array}\right] \\
& P_{22}=\frac{\gamma(s)}{d(s)}\left[\begin{array}{c}
k_{s}\left(m_{u} s^{2}+k_{t}\right) s^{2} \\
k_{s}\left(\left(m_{u}+m_{s}\right) s^{2}+k_{t}\right)
\end{array}\right]
\end{aligned}
$$

where $p_{1}(s)=m_{u} s^{2}+c_{s} s+k_{s}+k_{t}$ and

$$
\begin{aligned}
d(s)=\left(m_{s} m_{u}\right) s^{4}+c_{s}\left(m_{s}+m_{u}\right) s^{3} & \\
& +\left(m_{s}\left(k_{s}+k_{t}\right)+m_{u} k_{s}\right) s^{2}+c_{s} k_{t} s+k_{s} k_{t} .
\end{aligned}
$$

As expected, all roots of $d(s)$ are in left-half plane, which can be confirmed by the Routh-Hurwitz criterion.

We now observe that $P_{12,1}=P_{12}$, which has normal rank equal to one, i.e., $r_{2}=m_{3}$. Since $P$ is open-loop stable and $K_{0}=0$, the conditions of Theorem 2 are satisfied. We can then apply Theorem 2(2) to find the matrix $\widetilde{U}_{2}$ which defines the required control structure. Following the definitions in Theorem 2(2) we first find

$$
T_{3,1}=\tilde{M} P_{21,1}=\frac{k_{t} s^{2}}{d(s)}\left[\begin{array}{c}
c_{s} s+k_{s} \\
-m_{s}
\end{array}\right]
$$

which has normal rank equal to one, i.e., $r_{3}=1$. Hence we can select

$$
\begin{aligned}
F & =\frac{k_{t} s^{2}(s+\alpha)}{d(s)} \\
U_{1} & =\frac{1}{(s+\alpha)}\left[\begin{array}{c}
c_{s} s+k_{s} \\
-m_{s}
\end{array}\right]
\end{aligned}
$$

for any $\alpha>0$, to give a $r n f$ of $T_{3,1}$. We can choose $U_{2}$ to complete a unimodular matrix $U$ as follows:

$$
U=\left(U_{1}, U_{2}\right)=\left[\begin{array}{cc}
\frac{c_{s} s+k_{s}}{s+\alpha} & 0 \\
\frac{-m_{s}}{s+\alpha} & 1
\end{array}\right]
$$

which gives $U^{-1}=\left[\widetilde{U}_{1}^{\prime}, \widetilde{U}_{2}^{\prime}\right]^{\prime}$ and

$$
\widetilde{U}_{2}=\left[\frac{m_{s}}{c_{s} s+k_{s}} 1\right] \text {. }
$$

Thus, all stabilizing controllers which leave $T_{\hat{z}_{r} \rightarrow\left[\hat{z}_{s}, \hat{z}_{u}\right]^{\prime}}$ the same as in the open-loop can then be expressed as shown in Fig. 4 where

$$
K_{1}=-\left(I-Q_{1} \widetilde{U}_{2} P_{22}\right)^{-1} Q_{1}
$$

for $Q_{1} \in \mathbb{R} \mathbb{H}_{\infty}^{1 \times 1}$ or equivalently $K_{1}$ is any stabilizing controller for $\widetilde{U}_{2} P_{22}$ (see Theorem 2). 


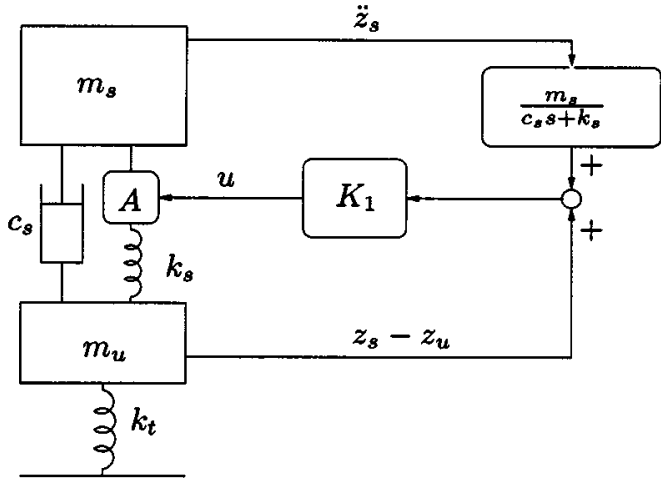

Fig. 4. Controller structure for the quarter-car with two feedbacks.

Example 1: We now apply the above result to a specific case in which we make the suspension stiff to load disturbances but soft to road disturbances. We select the following parameters for the quarter-car model as in [19] which correspond roughly to a small saloon car [3]: $m_{s}=250 \mathrm{~kg}, m_{u}=35 \mathrm{~kg}, k_{t}=$ $150 \mathrm{kN} / \mathrm{m}$. We choose $\omega_{n}=100 \mathrm{rad} / \mathrm{s}$ and $\delta=0.7071$ as the parameters for the actuator dynamics. We also choose $k_{s}=$ $12 \mathrm{kN} / \mathrm{m}, c_{s}=4 \mathrm{kNs} / \mathrm{m}$ as the spring-damper coefficients which we consider to give a suitable "soft" response from the road disturbances in the passive implementation. It is now required to design the active controller to achieve desirable responses from the load disturbances.

A simple approach is to take $Q_{1}$ to be constant in (20) and to minimize the steady-state response from load disturbances to sprung mass position. A straightforward calculation shows that $T_{\hat{F}_{s} \rightarrow \hat{z}_{s}}(0)=\left(k_{s}+k_{t}-Q_{1} k_{t}\right) /\left(k_{s} k_{t}\right)$ which can be made to equal zero when $Q_{1}=\left(k_{s}+k_{t}\right) / k_{t}=1.08$. The step responses for the passive and active suspensions are shown in Fig. 5, which clearly illustrates the zero steady-state response to loads achieved by the active controller. The following closed-loop eigenvalues were obtained: $-3.00,-3.95,-21.18 \pm 13.09 j$, $-83.97,-70.71 \pm 70.71 j$.

As a second approach we can employ the $H_{\infty}$ loop shaping design procedure [11], [30] to the plant $\widetilde{U}_{2} P_{22}$. We select a weighting function $W_{1}=10(s+80) /(s+8)$, so that the open-loop loop shape, $W_{1} \widetilde{U}_{2} P_{22}$, has a gain crossover frequency at about $44 \mathrm{rad} / \mathrm{s}$, somewhat below the actuator cutoff frequency of $100 \mathrm{rad} / \mathrm{s}$. The use of a lag compensator in $W_{1}$ allows the gain to be increased relatively at low frequencies in order to achieve a smaller value of $T_{\hat{F}_{s} \rightarrow \hat{z}_{s}}(0)$. This choice of weighting function gives a stability margin of 0.3864 . The final controller takes the form $K=K_{1} \widetilde{U}_{2}$ (see Fig. 4) where

$$
K_{1}=\frac{-23.87(s+25.37)(s+72.55 \pm 75.70 j)}{(s+8.10)(s+92.22 \pm 108.18 j)}
$$

which has been reduced to third order by balanced truncation. For this controller the step response from $F_{s}$ to $z_{s}$ is shown in Fig. 6, which exhibits an improved transient response in comparison to Fig. 5 but inferior steady-state behavior. The following closed-loop eigenvalues were obtained: -3.95 , $-21.18 \pm 13.09 j,-35.52 \pm 25.75 j,-83.97,-56.52 \pm 64.31 j$, $-76.44 \pm 93.06 j$.

At this point it is instructive to compare the control structure shown in Fig. 4 with a scheme presented by Williams $e t$
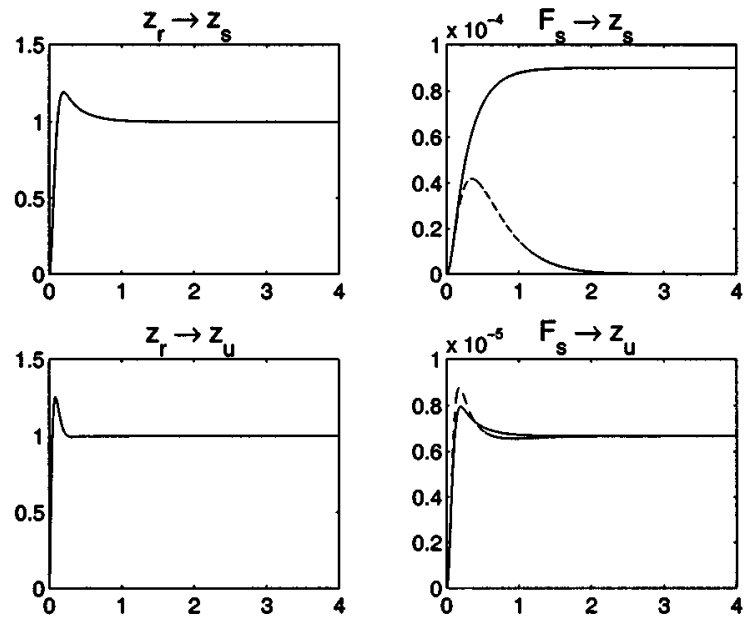

Fig. 5. Passive suspension $\left(Q_{1}=0\right.$, solid $)$ and active suspension $\left(Q_{1}=\right.$ 1.08 , dashed).

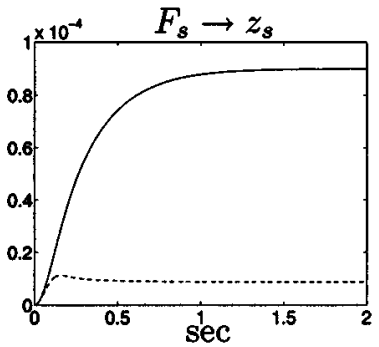

Fig. 6. Passive suspension (solid) and active suspension using $H_{\infty}$ loop shaping design (dashed).

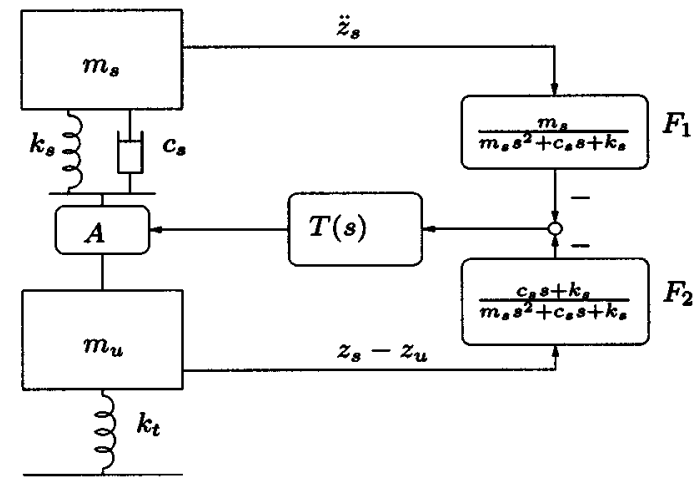

Fig. 7. Controller structure of Williams et al.

al. in [25]. The stated aim of their controller is to provide a rapid closed-loop levelling system which does not respond to unwanted road disturbances, and this is achieved "by filtering and summing the sprung mass acceleration and suspension displacement signals to eliminate the effects of the road inputs." A block diagram of the scheme in [25] is shown in Fig. 7 where $T(s)$ is a phase lead compensator. Although the damper is placed in series with the actuator this is not an essential difference. It may be observed that the ratio between the two filters $F_{1}$ and $F_{2}$ is equal to $m_{s} /\left(c_{s} s+k_{s}\right)$ so the scheme operates in a similar way to that of Fig. 4. In fact it can be shown that, if the denominators in $F_{1}$ and $F_{2}$ are both replaced by $(s+\alpha)$, and $T(s)$ is any stabilizing controller, then the scheme parameterizes all controllers which leave the 
road disturbance responses the same as in the open loop. Thus the scheme lacks full generality only by virtue of the fact that the filters $F_{1}$ and $F_{2}$ have an extra order of roll-off at high frequency, which is a minor difference since it may be useful to provide some high-frequency roll-off in $K_{1}$ in practice.

We point out that our chosen scheme, as well as the approach of [25], assumes that the ride performance is satisfactory with $K_{0}=0$. If this is not the case, a controller $K_{0} \neq 0$ may first be design to give any other desired road disturbance responses. Thereafter, Corollary 1 may be utilized to shape the load disturbance responses as well.

Finally, it is useful to comment on the full set of performance requirements that are usually considered in suspension design. In addition to the sprung mass position as a function of road disturbances, which can be analyzed with regard to driver comfort, there are also issues such as tire normal loads (i.e., tire deflection) and rattle space (i.e., strut deflection). It was shown in [7] that if the transfer function from $z_{r}$ to $z_{s}$ is determined, then there is no additional freedom left in the road disturbance transmission path, i.e., the transfer functions $T_{\hat{z}_{r} \rightarrow \hat{z}_{r}-\hat{z}_{u}}$ and $T_{\hat{z}_{r} \rightarrow \hat{z}_{s}-\hat{z}_{u}}$ can be deduced directly. A similar fact was shown in [18] for the load disturbance transmission path. Thus, in the above approach to active suspension design, it is assumed that for each disturbance transmission path that is being dealt with, all the relevant factors (e.g., comfort, tire loads, suspension deflection) are taken account of together.

\section{B. The Quarter-Car With Three Measurements}

We continue to illustrate our basic theory by considering the quarter-car model with the additional measurement $\ddot{z}_{u}$. We now write the system in the form of Fig. 1 with $y=\left[\ddot{z}_{s}, z_{s}-z_{u}, \ddot{z}_{u}\right]^{\prime}$ and all other variables the same as in Section III-A. The general plant of (1) then has $P_{11}, P_{12}$ the same as (16), (17) and

$$
\begin{aligned}
& P_{21}=\frac{1}{d(s)}\left[\begin{array}{cc}
k_{t}\left(c_{s} s+k_{s}\right) s^{2} & p_{1}(s) s^{2} \\
-k_{t}\left(m_{s} s^{2}\right) & \left(m_{u} s^{2}+k_{t}\right) \\
k_{t}\left(m_{s} s^{2}+c_{s} s+k_{s}\right) s^{2} & \left(c_{s} s+k_{s}\right) s^{2}
\end{array}\right] \\
& P_{22}=\frac{\gamma(s)}{d(s)}\left[\begin{array}{c}
k_{s}\left(m_{u} s^{2}+k_{t}\right) s^{2} \\
k_{s}\left(\left(m_{u}+m_{s}\right) s^{2}+k_{t}\right) \\
-k_{s} m_{s} s^{4}
\end{array}\right]
\end{aligned}
$$

where $d(s), p_{1}(s)$ are given in and before (18).

As before we wish to parameterize all controllers which leave the transmission path from the road disturbance to $z_{s}$ and $z_{u}$ the same as in the open-loop, i.e., with $u=0$, which assumes that $k_{s}$ and $c_{s}$ are chosen to give satisfactory responses for this transmission path. We can check that the conditions of Theorem 2 again hold, so that we can follow the procedure to obtain the required controller structure.

Following the definitions in Theorem 2(2) we find that

$$
T_{3,1}=\tilde{M} P_{21,1}=\frac{k_{t} s^{2}}{d(s)}\left[\begin{array}{c}
c_{s} s+k_{s} \\
-m_{s} \\
m_{s} s^{2}+c_{s} s+k_{s}
\end{array}\right]
$$

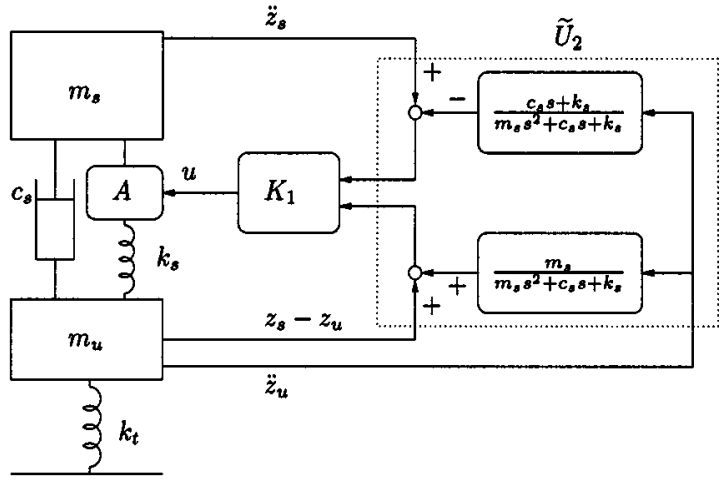

Fig. 8. Controller structure for the quarter-car with three feedbacks.

which gives $r_{3}=1$. Hence we can select

$$
\begin{aligned}
F & =\frac{k_{t} s^{2} p_{2}(s)}{d(s)}, \\
U_{1} & =\frac{1}{p_{2}(s)}\left[\begin{array}{c}
c_{s} s+k_{s} \\
-m_{s} \\
m_{s} s^{2}+c_{s} s+k_{s}
\end{array}\right]
\end{aligned}
$$

where $p_{2}(s)$ is any second-order Hurwitz polynomial, to give a $r n f$ of $T_{3,1}$. We can choose $U_{2}=\left[I_{2 \times 2}, 0_{2 \times 1}\right]^{\prime}$ to complete a unimodular matrix $U$ to give $U^{-1}=\left[\widetilde{U}_{1}^{\prime}, \widetilde{U}_{2}^{\prime}\right]$ with

$$
\widetilde{U}_{2}=\left[\begin{array}{ccc}
1 & 0 & -\frac{c_{s} s+k_{s}}{m_{s} s^{2}+c_{s} s+k_{s}} \\
0 & 1 & \frac{m_{s}}{m_{s} s^{2}+c_{s} s+k_{s}}
\end{array}\right] \text {. }
$$

Thus, all stabilizing controllers which leave $T_{\hat{z}_{r} \rightarrow\left[\hat{z}_{s}, \hat{z}_{u}\right]^{\prime}}$ the same as in the open-loop can then be expressed as shown in Fig. 8 where

$$
K_{1}=-\left(1-Q_{1} \widetilde{U}_{2} P_{22}\right)^{-1} Q_{1}
$$

for any $Q_{1} \in \mathbb{R} \mathbb{H}_{\infty}^{1 \times 2}$ or equivalently $K_{1}$ is any stabilizing controller for $\widetilde{U}_{2} P_{22}$ (see Theorem 2).

1) Alternative Controller Structures: As pointed out in Lemma 2 the exact form of $\widetilde{U}_{2}$ in (21) is not unique. Let us suppose that we prefer a structure with

$$
\widetilde{U}_{2}^{\dagger}=\left[\begin{array}{ccc}
f_{1} & 1 & 0 \\
0 & 1 & f_{2}
\end{array}\right]
$$

where $f_{1}, f_{2} \in \mathbb{R} \mathbb{H}_{\infty}$ are strictly proper, motivated by a preference to use low-pass filters for the acceleration signals $\ddot{z}_{s}$ and $\ddot{z}_{u}$ while keeping the strut deflection unfiltered. For $\widetilde{U}_{2}$ given by (21) the identity $\widetilde{U}_{2}^{\dagger}=W^{\dagger} \widetilde{U}_{2}$ implies that

$$
W^{\dagger}=\left[\begin{array}{cc}
\frac{m_{s}}{c_{s}+k_{s}} & 1 \\
0 & 1
\end{array}\right]
$$

and

$$
\widetilde{U}_{2}^{\dagger}=\left[\begin{array}{ccc}
\frac{m_{s}}{c_{s} s+k_{s}} & 1 & 0 \\
0 & 1 & \frac{m_{s}}{m_{s} s^{2}+c_{s} s+k_{s}}
\end{array}\right] .
$$

We can see that $W^{\dagger}$ fails to be unimodular as required by Theorem 2(3). Moreover, it is straightforward to show that the set $\left\{Q_{1}^{\dagger} \widetilde{U}_{2}^{\dagger}: Q_{1}^{\dagger} \in \mathbb{R} \mathbb{H}_{\infty}^{1 \times 2}\right\}$ is equal to the set $\left\{Q_{1} \widetilde{U}_{2}: Q_{1} \in \mathbb{R} \mathbb{H}_{\infty}^{1 \times 2}, Q_{1,1}(\infty)=0\right\}$, i.e., the full parameterization but with the first element in $Q_{1}$ being strictly proper. 
TABLE I

The $\widetilde{U}_{2}$ Structures AND the TRANSFormed Plants OF THE QuARTER-CAR MODEL With VARIOUS MEASUREMENTS

\begin{tabular}{c||c|c}
\hline measurements & $\tilde{U}_{2}$ & $\tilde{U}_{2} P_{22}$ \\
\hline$\left[\begin{array}{c}\ddot{z}_{s} \\
z_{s}-z_{u}\end{array}\right]$ & {$\left[\begin{array}{cc}\frac{m_{s}}{c_{s} s+k_{s}} & 1\end{array}\right]$} & $\frac{k_{s} \gamma(s)}{c_{s} s+k_{s}}$ \\
\hline$\left[\begin{array}{c}\ddot{z}_{s} \\
z_{s}-z_{u} \\
\ddot{z}_{u}\end{array}\right]$ & {$\left[\begin{array}{ccc}1 & 0 & -\frac{c_{s} s+k_{s}}{m_{s} s^{2}+c_{s} s+k_{s}} \\
0 & 1 & \frac{m_{s}}{m_{s} s^{2}+c_{s} s+k_{s}}\end{array}\right]$} & $\frac{k_{s} \gamma(s)}{m_{s} s^{2}+c_{s} s+k_{s}}\left[\begin{array}{c}s^{2} \\
1\end{array}\right]$ \\
\hline$\left[\begin{array}{c}\ddot{z}_{s} \\
z_{s}-z_{a} \\
z_{a}-z_{u}\end{array}\right]$ & {$\left[\begin{array}{ccc}0 & 1 & 0 \\
\frac{m_{s}}{c_{s} s+k_{s}} & 0 & 1\end{array}\right]$} & $\gamma(s)\left[\begin{array}{c}1 \\
\frac{-c_{s} s}{c_{s} s+k_{s}}\end{array}\right]$ \\
\hline
\end{tabular}

Thus a controller parameterization with $\widetilde{U}_{2}$ replaced by $\widetilde{U}_{2}^{\dagger}$ does not give all possible stabilizing controllers which leave the road disturbance responses the same as in the open-loop (see Lemma 2 and Theorem 2). However, the restriction of the $Q$-parameter amounts only to an increased roll-off requirement of the controller at high frequency.

Referring to Fig. 8, let us consider another possible controller structure

$$
\widetilde{U}_{2}^{+}=\left[\begin{array}{ccc}
f_{1} & 1 & 0 \\
1 & 0 & f_{2}
\end{array}\right]
$$

where $f_{1}, f_{2} \in \mathbb{R} \mathbb{H}_{\infty}$ are strictly proper. The identity $\widetilde{U}_{2}^{\ddagger}=$ $W+\widetilde{U}_{2}$ now implies that

$$
W^{+1}=\left[\begin{array}{cc}
\frac{m_{s}}{c_{s}+k_{s}} & 1 \\
1 & 0
\end{array}\right]
$$

and

$$
\widetilde{U}_{2}^{\ddagger}=\left[\begin{array}{ccc}
\frac{m_{s}}{c_{s} s+k_{s}} & 1 & 0 \\
1 & 0 & \frac{-\left(c_{s} s+k_{s}\right)}{m_{s} s^{2}+c_{s} s+k_{s}}
\end{array}\right] .
$$

Since $W^{\mathrm{t}}$ is unimodular, then $\widetilde{U}_{2}$ can be replaced by $\widetilde{U}_{2}^{\text {t }}$ in Fig. 8 to give a parameterization of all stabilizing controllers [Theorem 2(3)].

\section{Quarter-Car Control Structures and Design}

For direct controller design using Theorem 2, it is instructive to compute $\widetilde{U}_{2} P_{22}$ for the given choice of measurements. Table I shows $\widetilde{U}_{2} P_{22}$ for three different cases. It is interesting that $\widetilde{U}_{2} P_{22}$ takes a particularly simple form, which is independent of the sprung and unsprung masses, in the case when the feedback signals $\ddot{z}_{s}$ and $z_{s}-z_{u}$ are used, which means that the controller design would be rather simple in this case.

\section{THE HalF-CAR MODEL}

In this section, we shall apply the controller parameterization method to the half-car model shown in Fig. 9. As in the quarter-car model, the actuators $A_{1}$ and $A_{2}$ are modeled so that the relative displacement across each is equal to a low-pass filtered version of the actuator's command signal, i.e.,

$$
\begin{aligned}
& \hat{z}_{s}+l_{1} \hat{z}_{\psi}-\hat{z}_{a_{1}}=\gamma(s) \hat{u}_{1} \\
& \hat{z}_{s}-l_{2} \hat{z}_{\psi}-\hat{z}_{a_{2}}=\gamma(s) \hat{u}_{2}
\end{aligned}
$$

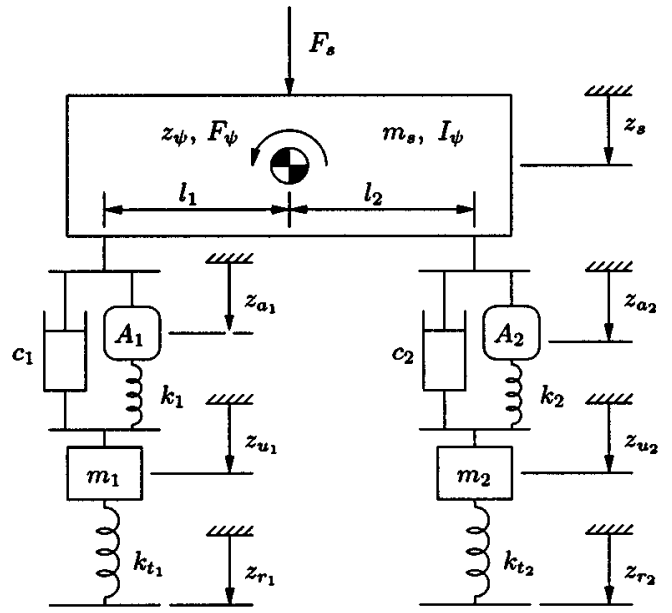

Fig. 9. The half-car model.

where $\gamma(s)$ is defined as in (11). The linearized dynamic equations can be expressed as follows:

$$
\begin{aligned}
m_{s} \ddot{z}_{s} & =F_{s}-u_{p_{1}}-u_{p_{2}} \\
I_{\psi} \ddot{z}_{\psi} & =F_{\psi}-u_{p_{1}} l_{1}+u_{p_{2}} l_{2} \\
m_{1} \ddot{z}_{u_{1}} & =u_{p_{1}}+F_{r_{1}} \\
m_{2} \ddot{z}_{u_{2}} & =u_{p_{2}}+F_{r_{2}}
\end{aligned}
$$

where the passive suspension forces $u_{p_{1}}, u_{p_{2}}$, and the tire forces $F_{r_{1}}, F_{r_{2}}$ are given by

$$
\begin{aligned}
& u_{p_{1}}=c_{1}\left(\dot{z}_{s}+l_{1} \dot{z}_{\psi}-\dot{z}_{u_{1}}\right)+k_{1}\left(z_{a_{1}}-z_{u_{1}}\right) \\
& u_{p_{2}}=c_{2}\left(\dot{z}_{s}-l_{2} \dot{z}_{\psi}-\dot{z}_{u_{2}}\right)+k_{2}\left(z_{a_{2}}-z_{u_{2}}\right) \\
& F_{r_{1}}=k_{t_{1}}\left(z_{r_{1}}-z_{u_{1}}\right) \\
& F_{r_{2}}=k_{t_{2}}\left(z_{r_{2}}-z_{u_{2}}\right) .
\end{aligned}
$$

We now write the system in the form of Fig. 1 with $z_{1}=\left[z_{s}, z_{\psi}, z_{u_{1}}, z_{u_{2}}\right]^{\prime}, w_{1}=\left[z_{r_{1}}, z_{r_{2}}\right]^{\prime}, w_{2}=\left[F_{s}, F_{\psi}\right]^{\prime}$, $y=\left[\ddot{z}_{s}, \ddot{z}_{\psi}, D_{1}, D_{2}\right]^{\prime}$ where $D_{1}=z_{s}+l_{1} z_{\psi}-z_{u 1}$, $D_{2}=z_{s}-l_{2} z_{\psi}-z_{u 2}$ are strut deflections, $u=\left[u_{1}, u_{2}\right]^{\prime}$ as in (23), (24) and $z_{2}$ omitted. As before we wish to parameterize all controllers which leave the transmission path from the road disturbances $\left[z_{r_{1}}, z_{r_{2}}\right]^{\prime}$ to $\left[z_{s}, z_{\psi}, z_{u_{1}}, z_{u_{2}}\right]^{\prime}$ the same as in the open-loop, i.e., with $u_{1}=u_{2}=0$, which assumes that $k_{1}$, $k_{2}, c_{1}$ and $c_{2}$ are chosen to give satisfactory responses for these transmission paths. We can check that the conditions of Theorem 2 again hold, so that we can follow the procedure to obtain the required controller structure.

Following the definitions in Theorem 2(2) we can choose a $U_{1}=T_{3,1} \times p_{3}(s) / s^{2}=P_{21,1} \times p_{3}(s) / s^{2} \in \mathbb{R} \mathbb{H}_{\infty}^{4 \times 2}$ and $F=s^{2} / p_{3}(s) \times I_{2 \times 2} \in \mathbb{R} \mathbb{H}_{\infty}^{2 \times 2}$, where $p_{3}(s)$ is any third-order Hurwitz polynomial, to give a $r n f$ of $T_{3,1}$, and complete this $U_{1}$ to a unimodular matrix with $U_{2}=\left[0_{2 \times 2}, I_{2 \times 2}\right]^{\prime}$ to give

$$
\widetilde{U}_{2}=\left[\begin{array}{cccc}
\frac{m_{s} l_{2}}{\left(c_{1} s+k_{1}\right)\left(l_{1}+l_{2}\right)} & \frac{I_{\psi}}{\left(c_{1} s+k_{1}\right)\left(l_{1}+l_{2}\right)} & 1 & 0 \\
\frac{m_{s} l_{1}}{\left(c_{2} s+k_{2}\right)\left(l_{1}+l_{2}\right)} & \frac{-I_{\psi}}{\left(c_{2} s+k_{2}\right)\left(l_{1}+l_{2}\right)} & 0 & 1
\end{array}\right] .
$$

We observe that $\widetilde{U}_{2}$ "constructs" two combinations of measurements, each of which is a suspension deflection plus a low-pass filtered version of a sum of the two acceleration signals. A block diagram of this control structure is shown in Fig. 10. 


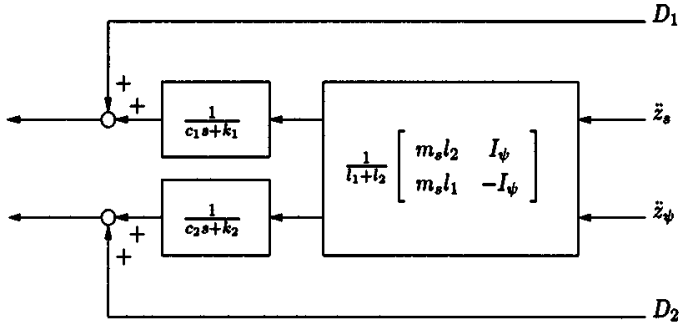

Fig. 10. Controller structure $\left(\widetilde{U}_{2}\right)$ for the half-car model.

For direct controller design using the transformed plant $P^{\dagger}$ in Fig. 2(b), it is interesting to note that $\widetilde{U}_{2} P_{22}$ takes a particularly simple form in the half-car case when $\widetilde{U}_{2}$ is determined by (29), namely

$$
\widetilde{U}_{2} P_{22}=\gamma(s)\left[\begin{array}{cc}
\frac{k_{1}}{c_{1}+k_{1}} & 0 \\
0 & \frac{k_{2}}{c_{2} s+k_{2}}
\end{array}\right] .
$$

This fact will be exploited in the design example for the full-car model in Section VI.

\section{A. Decoupling by Simplicity}

Under certain conditions, the half-car model can be structurally decoupled into two quarter-cars, and in such cases it is useful to exploit the simplified structure. In [19] and [22], assumptions such as $\mathrm{kl}$-simplicity were used, in a mechanical network setting, to perform energy-preserving transformations of the external disturbance variables to achieve decoupling. In our setting we will need to use a similar transformation on all of the system variables (but will not necessarily be able to respect the energy-preserving property). For the half-car model shown in Fig. 9, we define it as simple if the following equation holds:

$$
\frac{m_{1}}{m_{2}}=\frac{k_{1}}{k_{2}}=\frac{c_{1}}{c_{2}}=\frac{k_{t_{1}}}{k_{t_{2}}}=\frac{l_{2}}{l_{1}} .
$$

Note that half-car roll models are typically symmetric (i.e., $m_{1}=m_{2}$, etc.) so that (31) holds automatically in this case. Condition (31) may sometimes be satisfied also for half-car pitch models. We introduce a transformation matrix $L_{h}$ as follows:

$$
L_{h}=\frac{1}{l_{1}+l_{2}}\left[\begin{array}{cc}
l_{2} & l_{1} \\
1 & -1
\end{array}\right]
$$

and define

$$
\left[\begin{array}{l}
(x)_{b} \\
(x)_{\rho}
\end{array}\right]=L_{h}\left[\begin{array}{l}
x_{1} \\
x_{2}
\end{array}\right]
$$

where $x$ may represent any of the following variables: $z_{u}, z_{r}$, $z_{a}$, or $u$, and the subscripts $b$ and $\rho$ represent the bounce and rotation modes, respectively.

Under the assumption of simplicity, we can then rewrite (23)-(28) as follows:

$$
\begin{aligned}
\hat{z}_{s}-\left(\hat{z}_{a}\right)_{b}= & \gamma(s)(\hat{u})_{b} \\
\hat{z}_{\psi}-\left(\hat{z}_{a}\right)_{\rho}= & \gamma(s)(\hat{u})_{\rho} \\
m_{s} \ddot{z}_{s}= & F_{s}-\left(c_{1}+c_{2}\right)\left(\dot{z}_{s}-\left(\dot{z}_{u}\right)_{b}\right) \\
& -\left(k_{1}+k_{2}\right)\left(\left(z_{a}\right)_{b}-\left(z_{u}\right)_{b}\right) \\
I_{\psi} \ddot{z}_{\psi}= & F_{\psi}-\left(c_{1} l_{1}^{2}+c_{2} l_{2}^{2}\right)\left(\dot{z}_{\psi}-\left(\dot{z}_{u}\right)_{\rho}\right) \\
& +\left(k_{1} l_{1}^{2}+k_{2} l_{2}^{2}\right)\left(\left(z_{a}\right)_{\rho}-\left(z_{u}\right)_{\rho}\right)
\end{aligned}
$$

where $\gamma(s)$ is defined as in (11). The linearized dynamics of the full-car can be expressed as

$$
m_{s} \ddot{z}_{s}=F_{s}-u_{p_{1}}-u_{p_{2}}-u_{p_{3}}-u_{p_{4}}
$$

Comparing with (10), (12) and (13), we find that (33), (35) and (37) represent a bounce quarter-car, and (34), (36) and (38) represent a rotation quarter-car, which are decoupled from each other. The relevant correspondences between variables is summarized in Table II. Furthermore, we can show that, in order to arrive at a decoupled form for (35) and (36) (e.g., $z_{\psi}$ being absent from (35) etc), then we need both (31) to hold and for $L_{h}$ to be defined by (32) up to scalar multiplication of each row.

\section{THE Full-CAR Model}

In this section we shall introduce a standard full-car model with a similar suspension strut arrangement at each wheel-station to the quarter- and half-car cases in Figs. 3 and 9. We assume a left-right symmetry which allows a decoupling of the full-car model into two half-cars, namely the bounce/pitch and roll/warp half-cars. In preparation for a controller design in Section VI we will highlight the special form of the warp quarter-car, which has no "sprung mass dynamics."

\section{A. The Dynamic Equations}

Referring to Fig. 11, the actuators $A_{1}, \ldots, A_{4}$ are again modeled so that the relative displacement of each is equal to a low-pass filtered version of the actuator's command signals, i.e.,

$$
\begin{aligned}
& \hat{z}_{s}-l_{f} \hat{z}_{\theta}+t_{f} \hat{z}_{\phi}-\hat{z}_{a_{1}}=\gamma(s) \hat{u}_{1} \\
& \hat{z}_{s}-l_{f} \hat{z}_{\theta}-t_{f} \hat{z}_{\phi}-\hat{z}_{a_{2}}=\gamma(s) \hat{u}_{2} \\
& \hat{z}_{s}+l_{r} \hat{z}_{\theta}+t_{r} \hat{z}_{\phi}-\hat{z}_{a_{3}}=\gamma(s) \hat{u}_{3} \\
& \hat{z}_{s}+l_{r} \hat{z}_{\theta}-t_{r} \hat{z}_{\phi}-\hat{z}_{a_{4}}=\gamma(s) \hat{u}_{4}
\end{aligned}
$$

$m_{s} \ddot{z}_{s}=F_{s}-u_{p_{1}}-u_{p_{2}}-u_{p_{3}}-u_{p_{4}}$ 


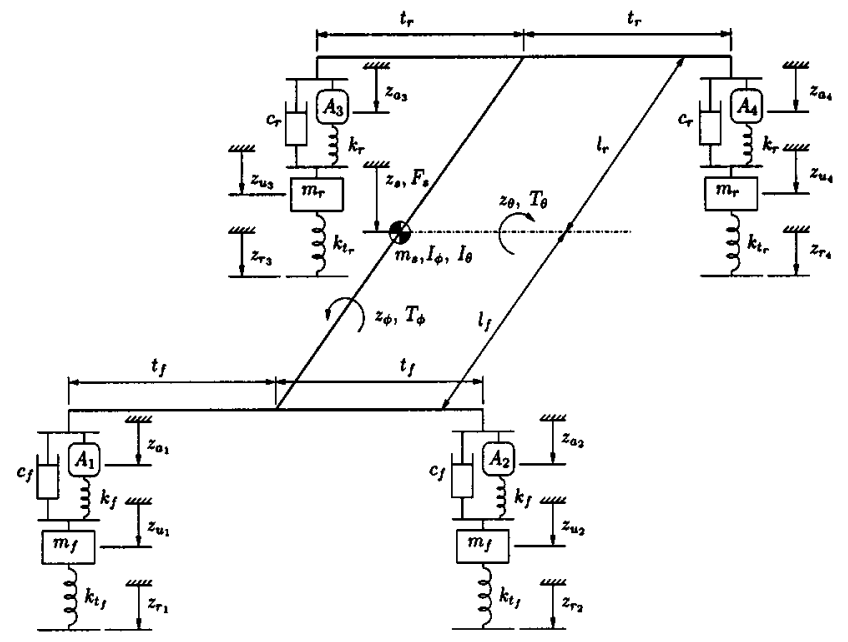

Fig. 11. The full-car model.

$$
\begin{aligned}
I_{\theta} \ddot{z}_{\theta} & =T_{\theta}+u_{p_{1}} l_{f}+u_{p_{2}} l_{f}-u_{p_{3}} l_{r}-u_{p_{4}} l_{r} \\
I_{\phi} \ddot{z}_{\phi} & =T_{\phi}-u_{p_{1}} t_{f}+u_{p_{2}} t_{f}-u_{p_{3}} t_{r}+u_{p_{4}} t_{r} \\
m_{f} \ddot{z}_{u_{1}} & =u_{p_{1}}+F_{r_{1}} \\
m_{f} \ddot{z}_{u_{2}} & =u_{p_{2}}+F_{r_{2}} \\
m_{r} \ddot{z}_{u_{3}} & =u_{p_{3}}+F_{r_{3}} \\
m_{r} \ddot{z}_{u_{4}} & =u_{p_{4}}+F_{r_{4}}
\end{aligned}
$$

where the passive suspension forces $u_{p_{1}}, \ldots, u_{p_{4}}$ and the tire forces $F_{r_{1}}, \ldots, F_{r_{4}}$ are given by

$$
\begin{aligned}
& u_{p_{i}}=c_{f} \dot{D}_{i}+k_{f}\left(z_{a_{i}}-z_{u_{i}}\right) \\
& u_{p_{j}}=c_{r} \dot{D}_{j}+k_{r}\left(z_{a_{j}}-z_{u_{j}}\right) \\
& F_{r_{i}}=k_{t_{f}}\left(z_{r_{i}}-z_{u_{i}}\right) \\
& F_{r_{j}}=k_{t_{r}}\left(z_{r_{j}}-z_{u_{j}}\right)
\end{aligned}
$$

for $i=1,2, j=3,4$, and the strut deflections $D_{1}, \ldots, D_{4}$ are

$$
\begin{aligned}
& D_{1}=z_{s}-l_{f} z_{\theta}+t_{f} z_{\phi}-z_{u_{1}} \\
& D_{2}=z_{s}-l_{f} z_{\theta}-t_{f} z_{\phi}-z_{u_{2}} \\
& D_{3}=z_{s}+l_{r} z_{\theta}+t_{r} z_{\phi}-z_{u_{3}} \\
& D_{4}=z_{s}+l_{r} z_{\theta}-t_{r} z_{\phi}-z_{u_{4}} .
\end{aligned}
$$

\section{B. Symmetric Transformation}

Since the full-car model is symmetric, we can decouple it into two half-car models. First, we introduce a transformation matrix $L_{f, 1}$ :

$$
L_{f, 1}=\frac{1}{2}\left[\begin{array}{cccc}
1 & 1 & 0 & 0 \\
0 & 0 & 1 & 1 \\
1 & -1 & 0 & 0 \\
0 & 0 & 1 & -1
\end{array}\right]
$$

such that

$$
\left[(x)_{b_{f}} \quad(x)_{b_{r}} \quad(x)_{\rho_{f}} \quad(x)_{\rho_{r}}\right]^{\prime}=L_{f, 1}\left[\begin{array}{llll}
x_{1} & x_{2} & x_{3} & x_{4}
\end{array}\right]^{\prime}
$$

where $x$ may represent any of the following variables: $z_{u}, z_{r}$, $z_{a}$, strut deflection $D$ or actuator command signal $u$, while the
TABLE III

DECOUPLED (SYMMETRIC) FULL-CAR

\begin{tabular}{l||l|l}
\hline Half-car & $\begin{array}{l}\text { Full-car } \\
\text { (bounce/pitch) }\end{array}$ & $\begin{array}{l}\text { Full-car } \\
\text { (roll/warp) }\end{array}$ \\
\hline$m_{s}$ & $m_{s}$ & - \\
$I_{\psi}$ & $I_{\theta}$ & $I_{\phi}$ \\
$m_{1}$ & $2 m_{f}$ & $2 m_{f}$ \\
$m_{2}$ & $2 m_{r}$ & $2 m_{r}$ \\
$k_{1}$ & $2 k_{f}$ & $2 k_{f}$ \\
$k_{2}$ & $2 k_{r}$ & $2 k_{r}$ \\
$c_{1}$ & $2 c_{f}$ & $2 c_{f}$ \\
$c_{2}$ & $2 c_{r}$ & $2 c_{r}$ \\
$k_{t_{1}}$ & $2 k_{t_{f}}$ & $2 k_{t_{f}}$ \\
$k_{t_{2}}$ & $2 k_{t_{r}}$ & $2 k_{t_{r}}$ \\
$l_{1}$ & $l_{f}$ & $t_{f}$ \\
$l_{2}$ & $l_{r}$ & $t_{r}$ \\
$F_{s}$ & $F_{s}$ & - \\
$F_{\psi}$ & $-T_{\theta}$ & $T_{\phi}$ \\
$z_{s}$ & $z_{s}$ & - \\
$z_{\psi}$ & $-z_{\theta}$ & $z_{\phi}$ \\
$z_{u_{1}}, z_{u_{2}}$ & $\left(z_{u}\right)_{b_{f}},\left(z_{u}\right)_{b_{r}}$ & $\left(z_{u}\right)_{\rho_{f}},-\left(z_{u}\right)_{\rho_{r}}$ \\
$z_{r_{1}}, z_{r_{2}}$ & $\left(z_{r}\right)_{b_{f}},\left(z_{r}\right)_{b_{r}}$ & $\left(z_{r}\right)_{\rho_{f}},-\left(z_{r}\right)_{\rho_{r}}$ \\
$z_{a_{1}}, z_{a_{2}}$ & $\left(z_{a}\right)_{b_{f}},\left(z_{a}\right)_{b_{r}}$ & $\left(z_{a}\right)_{\rho_{f}},-\left(z_{a}\right)_{\rho_{r}}$ \\
$u_{1}, u_{2}$ & $(u)_{b_{f}},(u)_{b_{r}}$ & $(u)_{\rho_{f}},-(u)_{\rho_{r}}$ \\
\hline
\end{tabular}

subscripts $_{b_{f}}, b_{r}$ represent the front and rear bounce components $\rho_{f}, \rho_{r}$ represent the front and rear roll components.

1) Bounce/Pitch Half-Car: After applying the $L_{f, 1}$ transformation, (39), (40), and (42)-(45) can be rearranged as follows:

$$
\begin{aligned}
m_{s} \ddot{z}_{s}-F_{s}= & -2 c_{f}\left(\dot{z}_{s}-l_{f} \dot{z}_{\theta}-\left(\dot{z}_{u}\right)_{b_{f}}\right) \\
& -2 k_{f}\left(\left(z_{a}\right)_{b_{f}}-\left(z_{u}\right)_{b_{f}}\right) \\
& -2 c_{r}\left(\dot{z}_{s}+l_{r} \dot{z}_{\theta}-\left(\dot{z}_{u}\right) b_{r}\right) \\
& -2 k_{r}\left(\left(z_{a}\right)_{b_{r}}-\left(z_{u}\right)_{b_{r}}\right), \\
I_{\theta} \ddot{z}_{\theta}-T_{\theta}= & \left\{2 c_{f}\left(\dot{z}_{s}-l_{f} \dot{z}_{\theta}-\left(\dot{z}_{u}\right)_{b_{f}}\right)\right. \\
& \left.+2 k_{f}\left(\left(z_{a}\right)_{b_{f}}-\left(z_{u}\right)_{b_{f}}\right)\right\} l_{f} \\
& -\left\{2 c_{r}\left(\dot{z}_{s}+l_{r} \dot{z}_{\theta}-\left(\dot{z}_{u}\right)_{b_{r}}\right)\right. \\
& \left.+2 k_{r}\left(\left(z_{a}\right)_{b_{r}}-\left(z_{u}\right)_{b_{r}}\right)\right\} l_{r} \\
2 m_{f}\left(\ddot{z}_{u}\right)_{b_{f}}= & 2 c_{f}\left(\dot{z}_{s}-l_{f} \dot{z}_{\theta}-\left(\dot{z}_{u}\right)_{b_{f}}\right) \\
& +2 k_{f}\left(\left(z_{a}\right)_{b_{f}}-\left(z_{u}\right)_{b_{f}}\right) \\
& +2 k_{t_{f}}\left(\left(z_{r}\right)_{b_{f}}-\left(z_{u}\right)_{b_{f}}\right) \\
= & 2 c_{r}\left(\dot{z}_{s}+l_{r} \dot{z}_{\theta}-\left(\dot{z}_{u}\right)_{b_{r}}\right) \\
& +2 k_{r}\left(\left(z_{a}\right)_{b_{r}}-\left(z_{u}\right)_{b_{r}}\right) \\
& +2 k_{t_{r}}\left(\left(z_{r}\right)_{b_{r}}-\left(z_{u}\right)_{b_{r}}\right) .
\end{aligned}
$$

It can be observed that the above equations take the same form as (25)-(28) under the transformations listed in the first column of Table III. To decouple into two quarter-cars requires a simplicity assumption

$$
\frac{k_{f}}{k_{r}}=\frac{c_{f}}{c_{r}}=\frac{k_{t_{f}}}{k_{t_{r}}}=\frac{m_{f}}{m_{r}}=\frac{l_{r}}{l_{f}} .
$$


TABLE IV

DECOUPLED ROLL AND WARP MODES OF THE FULL-CAR BY SIMPLICITY

\begin{tabular}{l||l|l}
\hline Quarter-car & Full-car (Warp) & Full-car (Roll) \\
\hline$m_{s}$ & - & $I_{\phi}$ \\
$m_{u}$ & $2\left(m_{f}+m_{r}\right)$ & $2\left(m_{f} t_{f}^{2}+m_{r} t_{r}^{2}\right)$ \\
$k_{s}$ & $2\left(k_{f}+k_{r}\right)$ & $2\left(k_{f} t_{f}^{2}+k_{r} t_{r}^{2}\right)$ \\
$c_{s}$ & $2\left(c_{f}+c_{r}\right)$ & $2\left(c_{f} t_{f}^{2}+c_{r} t_{r}^{2}\right)$ \\
$k_{t}$ & $2\left(k_{t_{f}}+k_{t_{r}}\right)$ & $2\left(k_{t_{f}} t_{f}^{2}+k_{t_{r}} t_{r}^{2}\right)$ \\
$F_{s}$ & - & $T_{\phi}$ \\
$z_{s}$ & - & $z_{\phi}$ \\
$z_{u}, z_{a}$ & $\left(z_{u}\right)_{\text {warp }},\left(z_{a}\right)_{\text {warp }}$ & $\left(z_{u}\right)_{\text {roll }},\left(z_{a}\right)_{\text {roll }}$ \\
$z_{r}, u$ & $\left(z_{r}\right)_{\text {warp }},(u)_{\text {warp }}$ & $\left(z_{r}\right)_{\text {roll }},(u)_{\text {roll }}$ \\
\hline
\end{tabular}

2) Roll/Warp Half-Car: After applying the $L_{f, 1}$ transformation, (41)-(45) can be rearranged as follows:

$$
\begin{aligned}
I_{\phi} \ddot{z}_{\phi}-T_{\phi}=- & \left\{2 c_{f}\left(t_{f} \dot{z}_{\phi}-\left(\dot{z}_{u}\right)_{\rho_{f}}\right)\right. \\
& \left.+2 k_{f}\left(\left(z_{a}\right)_{\rho_{f}}-\left(z_{u}\right) \rho_{f}\right)\right\} t_{f} \\
- & \left\{2 c_{r}\left(t_{r} \dot{z}_{\phi}-\left(\dot{z}_{u}\right)_{\rho_{r}}\right)\right. \\
& \left.+2 k_{r}\left(\left(z_{a}\right)_{\rho_{r}}-\left(z_{u}\right) \rho_{r}\right)\right\} t_{r} \\
2 m_{f}\left(\ddot{z}_{u}\right)_{\rho_{f}}= & 2 c_{f}\left(t_{f} \dot{z}_{\phi}-\left(\dot{z}_{u}\right)_{\rho_{f}}\right)+2 k_{f}\left(\left(z_{a}\right)_{\rho_{f}}-\left(z_{u}\right)_{\rho_{f}}\right) \\
& +2 k_{t_{f}}\left(\left(z_{r}\right)_{\rho_{f}}-\left(z_{u}\right)_{\rho_{f}}\right) \\
2 m_{r}\left(\ddot{z}_{u}\right)_{\rho_{r}}= & 2 c_{r}\left(t_{r} \dot{z}_{\phi}-\left(\dot{z}_{u}\right)_{\rho_{r}}\right)+2 k_{r}\left(\left(z_{a}\right)_{\rho_{r}}-\left(z_{u}\right)_{\rho_{r}}\right) \\
& +2 k_{t_{r}}\left(\left(z_{r}\right)_{\rho_{r}}-\left(z_{u}\right)_{\rho_{r}}\right) .
\end{aligned}
$$

We observe that one equation is missing in this half-car compared to (25)-(28). This is because the chassis is modeled as being infinitely stiff under torsion, so that there is no dynamic equation corresponding to warp dynamics of the car body. However the above three equations do take a similar form to (26)-(28) under the transformations listed in the second column of Table III.

As in the half-car case, under the assumption of simplicity

$$
\frac{k_{f}}{k_{r}}=\frac{c_{f}}{c_{r}}=\frac{k_{t_{f}}}{k_{t_{r}}}=\frac{m_{f}}{m_{r}}=\frac{t_{r}}{t_{f}}
$$

and the $L_{f, 2}$ transformation

$$
L_{f, 2}=\frac{1}{t_{f}+t_{r}}\left[\begin{array}{cc}
t_{r} & -t_{f} \\
1 & 1
\end{array}\right]
$$

such that

$$
\left[\begin{array}{c}
(x)_{\mathrm{warp}} \\
(x)_{\mathrm{roll}}
\end{array}\right]=L_{f, 2}\left[\begin{array}{l}
(x)_{\rho_{f}} \\
(x)_{\rho_{r}}
\end{array}\right]
$$

where $x$ can be $z_{u}, z_{r}$ or $z_{a}$, strut deflection $D$ or actuator command signal $u$, the roll/warp half-car can be further decoupled into roll and warp quarter-cars under the mapping illustrated in Table IV.

\section{A Design Example for the Full-Car Model}

In this section, we shall synthesize an active controller for a specific full-car model. As in Section V, the model is chosen to be left-right symmetric which allows a decoupling into the bounce/pitch and roll/warp half-cars. Our design approach for

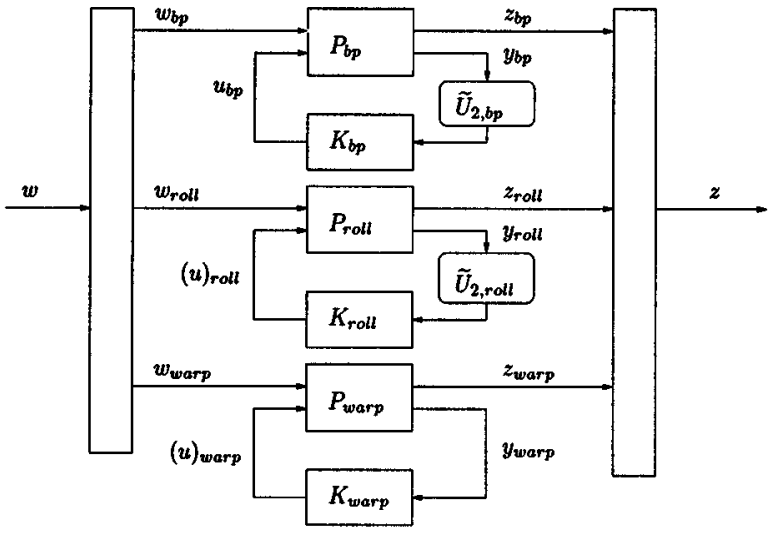

Fig. 12. Control scheme for the full-car model.

the bounce/pitch half-car will make use of the theory outlined in Section IV. The approach for the roll/warp half-car will make use of a simplicity assumption which allows it to be decoupled into the two corresponding quarter-cars, namely the roll and warp quarter-cars. The roll quarter-car will be treated in the same way as the quarter-car of Section III-A. As pointed out in Section V-B2, the warp quarter-car has a different form than the standard quarter-car in that the "sprung mass" is effectively infinite. Furthermore, in warp motion there is good reason to use the active controller to make the road disturbance responses even softer than they would be with the default passive parameter settings. Thus the warp mode will be handled in a different way to the other three modes.

For the controller design the available measurements are assumed to be $\ddot{z}_{s}, \ddot{z}_{\theta}, \ddot{z}_{\phi}, D_{1}, D_{2}, D_{3}$, and $D_{4}$. The control structure will be chosen to have three independent loops, consisting of the roll quarter-car, the warp quarter-car, and bounce/pitch half-car controllers. This scheme is shown in Fig. 12, where the signals are defined as follows:

$$
\begin{aligned}
w & =\left[F_{s}, T_{\theta}, T_{\phi}, z_{r_{1}}, z_{r_{2}}, z_{r_{3}}, z_{r_{4}}\right]^{\prime} \\
w_{b_{p}} & =\left[F_{s}, T_{\theta},\left(z_{r}\right)_{b_{f}},\left(z_{r}\right)_{b_{r}}\right]^{\prime} \\
w_{\text {roll }} & =\left[T_{\phi},\left(z_{r}\right)_{\mathrm{roll}}\right]^{\prime} \\
w_{\mathrm{warp}} & =\left[\left(z_{r}\right)_{\mathrm{warp}}\right] \\
z & =\left[z_{s}, z_{\theta}, z_{\phi}, z_{u_{1}}, z_{u_{2}}, z_{u_{3}}, z_{u_{4}}\right]^{\prime} \\
z_{b_{p}} & =\left[z_{s}, z_{\theta},\left(z_{u}\right)_{b_{f}},\left(z_{u}\right)_{b_{r}}\right]^{\prime} \\
z_{\text {roll }} & =\left[z_{\phi},\left(z_{u}\right)_{\mathrm{roll}}\right]^{\prime} \\
z_{\mathrm{warp}} & =\left[\left(z_{u}\right)_{\mathrm{warp}}\right] \\
y_{b p} & =\left[\ddot{z}_{s}, \ddot{z}_{\theta},(D)_{b_{f}},(D)_{b_{r}}\right]^{\prime} \\
y_{\mathrm{roll}} & =\left[\ddot{z}_{\phi},(D)_{\mathrm{roll}}\right] \\
y_{\mathrm{warp}} & =\left[(D)_{\mathrm{warp}}\right] \\
u_{b p} & =\left[(u)_{b_{f}},(u)_{b_{r}}\right]^{\prime}
\end{aligned}
$$

and the subscripts $b_{1}, b_{2}$, roll, and warp are defined as in (49) and (53).

The following parameters will be used for the full-car model which are similar to typical parameters for a sports car [3]: $m_{s}=$ $1600 \mathrm{~kg}, I_{\theta}=1000 \mathrm{~kg} \mathrm{~m}^{2}, I_{\phi}=450 \mathrm{~kg} \mathrm{~m}^{2}, t_{f}=t_{r}=0.75 \mathrm{~m}$, $l_{f}=1.15 \mathrm{~m}, l_{r}=1.35 \mathrm{~m}, k_{f}=k_{r}=20 \mathrm{kN} / \mathrm{m}, c_{f}=c_{r}=$ $5 \mathrm{kNs} / \mathrm{m}, m_{f}=m_{r}=50 \mathrm{~kg}, k_{t_{f}}=k_{t_{r}}=250 \mathrm{kN} / \mathrm{m}$. As in 

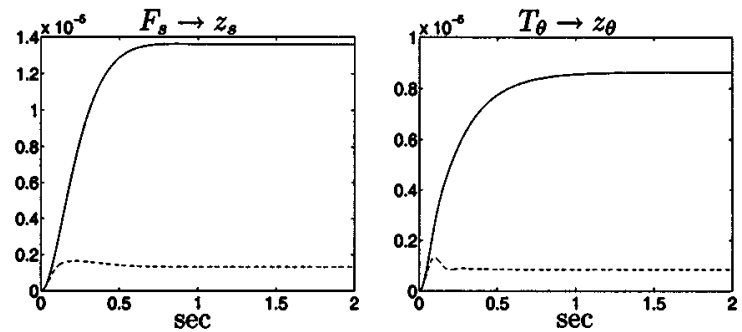

Fig. 13. Step responses of $T_{\hat{F}_{S} \rightarrow \hat{z}_{S}}$ and $T_{\hat{T}_{\theta} \rightarrow \hat{z}_{\theta}}$ : passive (solid) and active control (dashed).

the previous examples, the actuator dynamics is represented as in (11) with $\omega_{n}=100 \mathrm{rad} / \mathrm{s}$ and $\delta=0.707$.

\section{A. Bounce/Pitch Control}

Referring to Table III, the bounce/pitch half-car corresponds to the half-car of Section IV with the following coefficients: $m_{s}=1600 \mathrm{~kg}, I_{\psi}=1000 \mathrm{~kg} \mathrm{~m}^{2}, m_{1}=m_{2}=100 \mathrm{~kg}$, $k_{1}=k_{2}=40 \mathrm{kN} / \mathrm{m}, c_{1}=c_{2}=10 \mathrm{kNs} / \mathrm{m}, k_{t_{1}}=k_{t_{2}}=$ $500 \mathrm{kN} / \mathrm{m}, l_{1}=1.15 \mathrm{~m}, l_{2}=1.35 \mathrm{~m}$, which is not simple and cannot be decoupled into two quarter-cars. The $H_{\infty}$ loop shaping controller design will be applied to this half-car model. The essential controller structure $\widetilde{U}_{2, b p}$ is given by (29) as

$$
\widetilde{U}_{2, b p}=\left[\begin{array}{cccc}
\frac{m_{s} l_{r}}{2\left(c_{f} s+k_{f}\right)\left(l_{r}+l_{f}\right)} & \frac{m_{\theta}}{2\left(c_{f} s+k_{f}\right)\left(l_{r}+l_{f}\right)} & 1 & 0 \\
\frac{m_{s} l_{f}}{2\left(c_{r} s+k_{r}\right)\left(l_{r}+l_{f}\right)} & \frac{-I_{\theta}}{2\left(c_{r} s+k_{r}\right)\left(l_{r}+l_{f}\right)} & 0 & 1
\end{array}\right] \text {. }
$$

Setting a weighting function $W_{b p}$ as follows:

$$
W_{b p}=\left[\begin{array}{cc}
10 & 0 \\
0 & 10
\end{array}\right] \frac{s+80}{s+8}
$$

means that the weighted plant $W_{b p} \widetilde{U}_{2, b p} P_{22}$ has a bandwidth of about $60 \mathrm{rad} / \mathrm{s}$ and has an increased low-frequency gain due to the lag compensator terms. By applying the $H_{\infty}$ loop shaping controller design procedure a sixth-order controller was obtained after balanced truncation as follows:

$$
K_{b p}=\left[\begin{array}{cc}
K_{c} & 0 \\
0 & K_{c}
\end{array}\right]
$$

where

$$
K_{c}=\frac{-24.81(s+27.94)(s+72.56 \pm 73.47 j)}{(s+8.06)(s+101.45 \pm 114.65 j)} .
$$

It is interesting to note that $K_{b p}$ in (58) is a scalar matrix due to the fact that $W_{b p} \widetilde{U}_{2, b p} P_{22}$ is itself scalar (see (30)). This controller gives the dc gains $T_{\hat{F}_{s} \rightarrow \hat{z}_{s}}(0)$ and $T_{\hat{T}_{\theta} \rightarrow \hat{z}_{\theta}}(0)$ as $1.32 \times 10^{-6}$ and $8.39 \times 10^{-7}$, respectively, compared with $1.36 \times 10^{-5}$ and $8.64 \times 10^{-6}$ using passive control. The step responses using the two controllers are shown in Fig. 13.

\section{B. Roll Control}

Referring to Table IV, the roll mode of the full-car corresponds to a quarter-car with the following coefficients: $m_{s}=$ $450 \mathrm{~kg}, m_{u}=112.5 \mathrm{~kg}, k_{s}=45 \mathrm{kN} / \mathrm{m}, c_{s} \equiv 11.25 \mathrm{kNs} / \mathrm{m}$, $k_{t}=562.5 \mathrm{kN} / \mathrm{m}$. The required structure of $\widetilde{U}_{2}$ takes the following form after using Table IV, (19), and (51)

$$
\widetilde{U}_{2, \text { roll }}=\left[\begin{array}{ll}
\frac{I_{\phi}}{2\left(c_{f} s+k_{f}\right) t_{f}\left(t_{r}+t_{f}\right)} & 1
\end{array}\right] .
$$

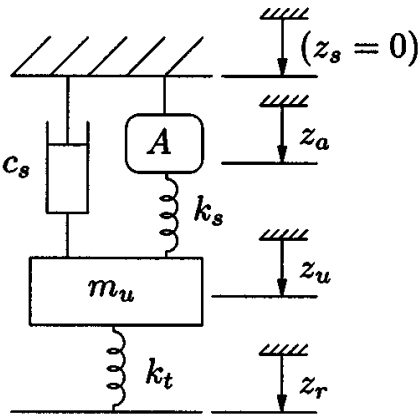

Fig. 14. The warp quarter-car.

Given the weighting function

$$
W_{\mathrm{roll}}=\frac{10(s+80)}{(s+8)}
$$

such that the weighted plant $W_{\text {roll }} \widetilde{U}_{2 \text {,roll }} P_{22}$ has a bandwidth of about $60 \mathrm{rad} / \mathrm{s}$, it is found that the $H_{\infty}$ loop shaping controller after model reduction is given by

$$
K_{\text {roll }}=K_{c}
$$

where $K_{c}$ is given by (59). (This controller is the same as the diagonal terms in $K_{b p}$ since the weighted plant $W_{\text {roll }} \widetilde{U}_{2 \text {,roll }} P_{22}$ is the same as the diagonal elements in the scalar matrix $W_{b p} \widetilde{U}_{2, b p} P_{22}$, see (30) and Table I.) This controller gives the steady state gain of $T_{\hat{T}_{\phi} \rightarrow \hat{z}_{\phi b}}$ as $2.33 \times 10^{-6}$, compared to the passive suspension with dc gain $T_{\hat{T}_{\phi} \rightarrow \hat{z}_{\phi}}(0)=2.4 \times 10^{-5}$, a similar result to Fig. 6.

\section{Warp Control}

For the warp quarter-car, we will take a slightly different approach for the design of the active controller. Since the sprung mass cannot be twisted, i.e., it has no warp motion, there is no corresponding role for the active controller to make the "sprung mass" stiffer to the loads. On the other hand, even though the passive road disturbance responses were designed to be relatively soft, there is no reason why they should not be even softer in the warp mode. We will therefore abandon the goal of keeping the response to the road warp input $\left(z_{r}\right)_{\text {warp }}$ invariant under active control. We also note that there is no acceleration measurement associated with warp and so there is only one feedback signal available corresponding to the strut deflections: $(D)_{\text {warp }}=-\left(z_{u}\right)_{\text {warp }}$. For this reason there is no $\widetilde{U}_{2}$ block for the warp quarter-car loop in Fig. 12.

Referring to Table IV, the warp quarter-car reduces to the form illustrated in Fig. 14, with $z_{s}=0$ and the coefficients $m_{u}=200 \mathrm{~kg}, k_{s}=80 \mathrm{kN} / \mathrm{m}, c_{s}=20 \mathrm{kNs} / \mathrm{m}$ and $k_{t}=$ $1000 \mathrm{kN} / \mathrm{m}$. The dynamic equation then takes the form, using (13)-(15)

$$
m_{u} s^{2} \hat{z}_{u}=-\left(c_{s} s+k_{s}\right) \hat{z}_{u}+k_{s} \hat{z}_{a}+k_{t}\left(\hat{z}_{r}-\hat{z}_{u}\right)
$$

which reduces to, using (10) and (11)

$$
\left(m_{u} s^{2}+c_{s} s+k_{s}+k_{t}-k_{s} \gamma(s) K_{\text {warp }}(s)\right) \hat{z}_{u}=k_{t} \hat{z}_{r}
$$

with the correspondences given in Table IV.

We now claim that it is desirable to choose $K_{\text {warp }}(s)$ so that the dc gain $T_{\hat{z}_{r} \rightarrow \hat{z}_{u}}(0)=1$. We will now give some reasoning 
to justify this. If we consider the case where the full-car model is in equilibrium with $F_{s}=T_{\theta}=T_{\phi}=0$, then the (39)-(41) are equivalent to

$$
\left[\begin{array}{cccc}
1 & 1 & 1 & 1 \\
l_{f} & l_{f} & -l_{r} & -l_{r} \\
-t_{f} & t_{f} & -t_{r} & t_{r}
\end{array}\right]\left[\begin{array}{l}
u_{p_{1}} \\
u_{p_{2}} \\
u_{p_{3}} \\
u_{p_{4}}
\end{array}\right]=0 .
$$

Evidently there is one degree of freedom available in the suspension forces, and indeed we can check that

$$
\left[u_{p_{1}}, u_{p_{2}}, u_{p_{3}}, u_{p_{4}}\right]^{\prime}=\left[t_{r},-t_{r},-t_{f}, t_{f}\right]^{\prime} \kappa
$$

for some constant $\kappa$, completely characterizes that freedom. From the point of view of reducing the amount of "twist" on the vehicle chassis which the suspension forces impose, it would be desirable to achieve a value of $\kappa=0$ in the steady state. The following result shows that the above mentioned condition achieves this property.

Proposition 1: Suppose the (linearized) full-car model defined in Section V-A is in equilibrium with $F_{s}=T_{\theta}=T_{\phi}=0$ and $z_{r_{1}}, \ldots, z_{r_{4}}$ arbitrary. Then the following equation holds:

$$
\left(z_{r}\right)_{\mathrm{warp}}=\left(z_{u}\right)_{\mathrm{warp}}
$$

if and only if $u_{p_{1}}=u_{p_{2}}=u_{p_{3}}=u_{p_{4}}=0$.

Proof: Using (49) and (53), we notice that the warp variable $(x)_{\text {warp }}$ is a combination of variables at the four wheelstations

$$
(x)_{\mathrm{warp}}=\frac{1}{2\left(t_{r}+t_{f}\right)}\left[t_{r},-t_{r},-t_{f}, t_{f}\right]\left[\begin{array}{l}
x_{1} \\
x_{2} \\
x_{3} \\
x_{4}
\end{array}\right]
$$

where $x$ can be $z_{u}$ or $z_{r}$. Using (46) and (47) we see that (66) is equivalent to $\left[t_{r} / k_{t_{f}},-t_{r} / k_{t_{f}},-t_{f} / k_{t_{r}}, t_{f} / k_{t_{r}}\right] \cdot\left[F_{r_{1}}, F_{r_{2}}, F_{r_{3}}, F_{r_{4}}\right]^{\prime}=0$, which is equivalent to $\left[t_{r} / k_{t_{f}},-t_{r} / k_{t_{f}},-t_{f} / k_{t_{r}}, t_{f} / k_{t_{r}}\right]$. $\left[u_{p_{1}}, u_{p_{2}}, u_{p_{3}}, u_{p_{4}}\right]^{\prime}=0$ by (42)-(45), which in turn is equivalent to $\kappa=0$ by (65).

It can be observed that the proposition holds if (66) is replaced by any equation of the form:

$$
\begin{aligned}
& {[\lambda,-\lambda,-\mu, \mu]} \\
& \cdot\left[z_{r_{1}}-z_{u_{1}}, z_{r_{2}}-z_{u_{2}}, z_{r_{3}}-z_{u_{3}}, z_{r_{4}}-z_{u_{4}}\right]^{\prime}=0
\end{aligned}
$$

with $\lambda \mu>0$, i.e., the particular ratios chosen in the definition of the warp variable are not critical to the result.

Now let us return to the warp quarter-car represented in (63). If we choose a simple constant controller and ignore temporarily the actuator dynamics, i.e., set $\gamma(s)=1$, then the choice of $K_{\text {warp }}=7.25$ achieves a damping ratio of one, a natural frequency equal to $50 \mathrm{rad} / \mathrm{s}$ (which is lower than the bandwidth of the actuator) and a steady-state gain of 2 . As shown by Proposition 1 we would like to achieve the condition (66) in the steadystate, which is equivalent to the dc gain $T_{\hat{z}_{r} \rightarrow \hat{z}_{u}}(0)=1$. Setting $K_{\text {warp }}(s)=-0.1036 s+1$ achieves in (63) a damping ratio of one, a natural frequency equal to $70.71 \mathrm{rad} / \mathrm{s}$ (which is lower

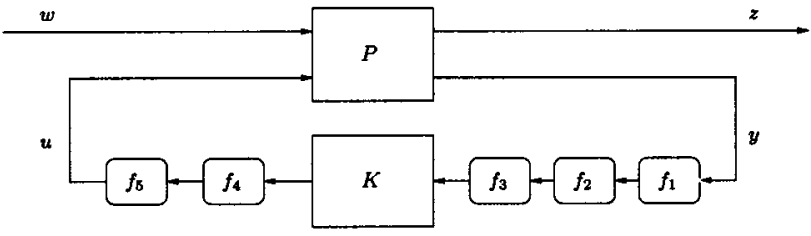

Fig. 15. Final controller structure for the full-car model.
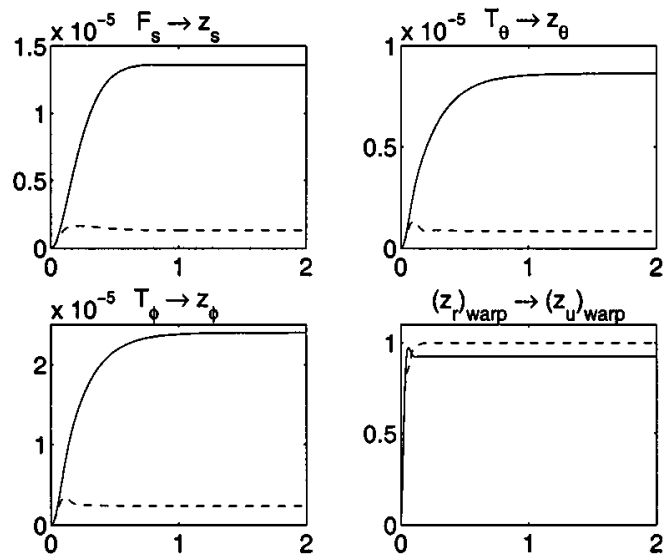

Fig. 16. Step responses using active (dashed) and passive (solid) suspensions.

than the bandwidth of the actuator) and a steady-state gain of one. In order that the controller is proper, we can choose

$$
K_{\text {warp }}=\frac{-0.1036 s+1}{(300)^{-1} s+1} .
$$

The response does not change significantly with this modification or when the actuator dynamics are included. The step response with the final controller, with improved warp behavior compared to the passive case, is shown in Fig. 16.

\section{The Full-Car Control}

As a final step we can redraw Fig. 12 in the form of Fig. 15 where $P$ is the full-car model represented by (39)-(45). In Fig. 15 the measurements $y$ and control signals $u$ are defined as follows:

$$
\begin{aligned}
y & =\left[\ddot{z}_{s}, \ddot{z}_{\theta}, \ddot{z}_{\phi}, D_{1}, D_{2}, D_{3}, D_{4}\right]^{\prime} \\
u & =\left[u_{1}, u_{2}, u_{3}, u_{4}\right]^{\prime}
\end{aligned}
$$

where $D_{i}$ and $u_{i}$ represent the strut deflection and control command signal at each wheel station. The blocks $f_{1}, \ldots, f_{5}$ are defined as follows:

$$
\begin{aligned}
f_{1} & =\operatorname{diag}\left(1,1,1, L_{f, 1}\right) \\
f_{2} & =\operatorname{diag}\left(1,-1,1,1,1, L_{f, 2}\right) \\
f_{3}= & {\left[\begin{array}{ccccccc}
\frac{m_{s} l_{r}}{d_{1}(s)} & \frac{I_{\theta}}{d_{1}(s)} & 0 & 1 & 0 & 0 & 0 \\
\frac{m_{s} l_{f}}{d_{2}(s)} & \frac{-I_{\theta}}{d_{2}(s)} & 0 & 0 & 1 & 0 & 0 \\
0 & 0 & 0 & 0 & 0 & 1 & 0 \\
0 & 0 & \frac{I_{\phi}}{d_{3}(s)} & 0 & 0 & 0 & 1
\end{array}\right] } \\
f_{4}= & \operatorname{diag}\left(1,1, L_{f, 2}^{-1}\right) \\
f_{5} & =L_{f, 1}^{-1}
\end{aligned}
$$

where $L_{f, 1}$ is defined in (48), $L_{f, 2}$ is defined in (52) and $d_{1}(s)=$ $2\left(c_{f} s+k_{f}\right)\left(l_{r}+l_{f}\right), d_{2}(s)=2\left(c_{r} s+k_{r}\right)\left(l_{r}+l_{f}\right), d_{3}(s)=$ 


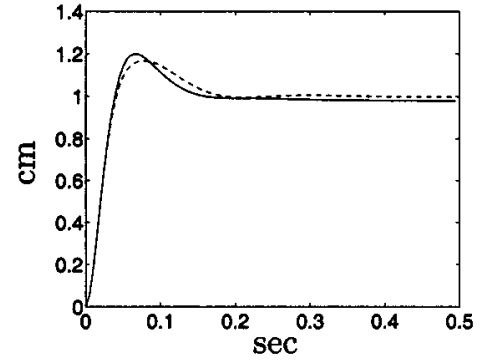

Fig. 17. Response of $z_{u_{1}}$ to a step input of $1 \mathrm{~cm}$ at $z_{r_{1}}$ for AutoSim model using active (dashed) and passive (solid) suspension.

$2\left(c_{f} s+k_{f}\right) t_{f}\left(t_{r}+t_{f}\right)$. Note that $f_{3}$ combines $\widetilde{U}_{2, b p}$ defined in (56) and $\widetilde{U}_{2}$,roll defined in (60), and the third row in $f_{3}$ reflects the fact that there is only one measurement available for warp control. The controller $K$ is defined as

$$
K=\operatorname{diag}\left(K_{b p}, K_{\text {warp }}, K_{\text {roll }}\right)
$$

where $K_{b p}, K_{\text {warp }}$ and $K_{\text {roll }}$ are given by (58), (62) and (67), respectively. Compared with the passive suspension, the benefits of using active controllers is shown in Fig. 16. The responses to "bounce," "pitch," and "roll" road inputs are not shown since these are the same in the passive and active cases.

\section{E. Vehicle Dynamics Simulations}

In this section we present some simulation results for the controller designed in Section VI-D using the multibody simulation package AutoSim. A nonlinear dynamical model of the simple full-car shown in Fig. 11 was constructed with the suspension struts constrained to move perpendicularly to the vehicle body. To model a rolling wheel of inertia $1 \mathrm{~kg} \mathrm{~m}^{2}$ with tire the magic formula [1] was employed to calculate the accelerating and braking forces. The control law given in Section VI-D was implemented together with the actuator structure described in Section V-A.

The model was first tested at zero velocity for various road disturbance inputs and gave similar results, for small displacements, to a Matlab simulation of the linearized model. As expected, the bounce, pitch and roll responses were the same in the active and passive cases. Fig. 17 shows the effect of applying a step input to the AutoSim model at the right front wheel in both the passive and active cases. The difference in behavior is due to the "warp" mode being treated differently in the active case, as explained in Section VI-C.

The AutoSim model was then tested under acceleration and braking. For acceleration, a torque was applied at each front wheel with the opposing reaction torques acting on the vehicle body. A similar approach was taken for braking but with the braking torques applied to the front and rear wheels in a 60:40 ratio. Fig. 18 shows the "squat" and "dive" of the model under acceleration and deceleration, with the forward velocity given in Fig. 18(a) and the pitch angle $z_{\theta}$ given in Fig. 18(c). The simulation shows that the active suspension significantly improves the squat and dive performance.

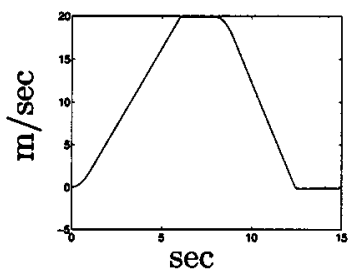

forward velocity

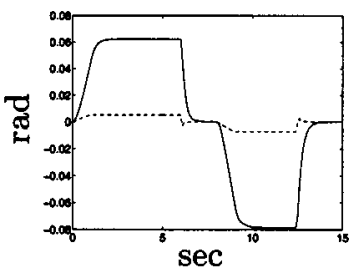

$z_{\theta}$

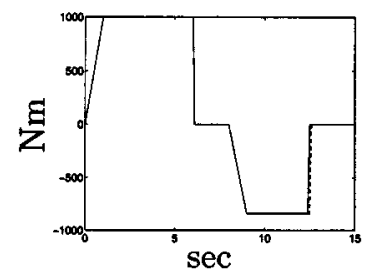

Applied torques on front wheels

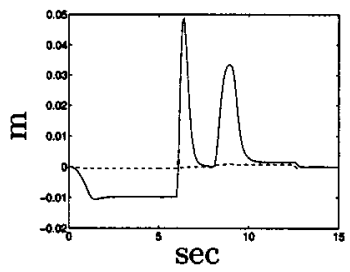

$z_{s}$
Fig. 18. Antidive and antisquat effect in AutoSim model using active suspension (dashed), compared with passive suspension (solid). (a) Forward velocity. (b) Applied torque on front wheels. (c) $z_{\theta}$. (d) $z_{s}$.

\section{CONCLUDING REMARKS}

This paper has considered the vehicle active suspension design problem with particular regard for the potentially conflicting performance requirements from two disturbance sources: road irregularities and loads applied to the vehicle body. General theorems (Theorems 1, 2) were derived to parameterize all stabilizing controllers which leave some prespecified closed-loop transfer function fixed. This allowed a feedback controller to be designed taking account of only the load disturbance path objective, given that the controller structure ensured that the road disturbance responses remained satisfactory.

The approach was illustrated for the quarter-car model with various different choices of measurements. The required control structures were derived in parametric form. For a half-car model, a parametric control structure was derived for a typical measurement set: vertical and angular accelerations of the sprung mass and strut deflection measurements. The conditions under which the model structure could be decomposed into two quarter-cars was investigated. For the full-car model, decomposition into two half-cars was exploited under a mild symmetry assumption. This enabled the bounce/pitch half-car design to be carried out with the half-car structure previously derived. For the roll/warp half-car a further decomposition into two quarter-cars was assumed. This allowed the warp quarter-car to be treated in a distinct way, which is necessary since the load disturbance path is absent here and it is also reasonable to change (i.e., soften) the road disturbance response from the passive case. A controller was designed and demonstrated on a nonlinear vehicle dynamics model and showed the effectiveness of the design for reduced dive and squat under acceleration and braking, improved warp response and invariance of other road disturbance responses. 
A key step in the method described in this paper is the computation of the matrix $\widetilde{U}_{2}$ which determines the required controller structure. Throughout the paper it was always possible to calculate $\widetilde{U}_{2}$ symbolically using Maple. For more complicated vehicle models this may not be feasible. In such a case a direct numerical approach may be possible. Let us consider the case $r_{3}=m_{1}$, i.e., the matrix $F$ in (6) is square. (This condition applied throughout this paper and seems quite typical in general.) Then the following procedure can be taken: 1) partition $T_{3,1}=\left[B^{\prime}, A^{\prime}\right]^{\prime}$ where $B$ is square; 2) find a minimal realization of $L:=A B^{-1}$; and 3) find a left coprime factorization $L=\widetilde{B}^{-1} \widetilde{A}$ and set $\widetilde{U}_{2}=[-\widetilde{A}, \widetilde{B}]$ (e.g., see [30, Theorem 12.19]. Such an approach was taken in a trailing-arm vehicle model in [23].

\section{APPENDIX}

Proof of Lemma 1: $T$ can be decomposed in terms of its Smith form over $\mathbb{R} \mathbb{H}_{\infty}$ [21]: $T=U H V$, where $U \in \mathbb{R} \mathbb{R}_{\infty}^{r \times r}$, $V \in \mathbb{R} \mathbb{H}_{\infty}^{c \times c}$ are unimodular, $H \in \mathbb{R} \mathbb{H}_{\infty}^{r \times c}$. Suppose that $H$ has $r_{1}$ nonzero diagonal elements. Then we can write

$$
\begin{aligned}
H & =\left(\begin{array}{c}
H_{1} \\
0_{\left(r-r_{1}\right) \times r_{1}}
\end{array}\right)\left(I_{r_{1} \times r_{1}}, 0_{r_{1} \times\left(c-r_{1}\right)}\right) \\
& =\left(\begin{array}{c}
I_{r_{1} \times r_{1}} \\
0_{\left(r-r_{1}\right) \times r_{1}}
\end{array}\right)\left(H_{1}, 0_{r_{1} \times\left(c-r_{1}\right)}\right)
\end{aligned}
$$

where $H_{1} \in \mathbb{R} \mathbb{H}_{\infty}^{r_{1} \times r_{1}}$. We also partition $U$ and $V$ conformably: $U=\left(U_{1}, U_{2}\right), V=\left(V_{1}^{\prime}, V_{2}^{\prime}\right)^{\prime}$ where $U_{1} \in \mathbb{R} \mathbb{R}_{\infty}^{r \times r_{1}}, U_{2} \in$ $\mathbb{R} \mathbb{R} \mathbb{W}_{\infty}^{r \times\left(r-r_{1}\right)}, V_{1} \in \mathbb{R} \|_{\infty}^{r_{1} \times c}, V_{2} \in \mathbb{R} \mathbb{U}_{\infty}^{\left(c-r_{1}\right) \times c}$. We therefore obtain

$$
\begin{aligned}
T & =\left(U_{1} H_{1}\right) V_{1}=E V_{1} \\
& =U_{1}\left(H_{1} V_{1}\right)=U_{1} F
\end{aligned}
$$

where $E \in \mathbb{R} \mathbb{R}_{\infty}^{r \times r_{1}}, F \in \mathbb{R} \prod_{\infty}^{r_{1} \times c}$ are full column normal rank and full row normal rank, respectively.

Proof of Theorem 1: A stabilizing controller in the form (3) leaves $T_{\hat{w}_{1} \rightarrow \hat{z}_{1}}=\left(T_{1}\right)_{11}$ if and only if $\left(T_{2} Q T_{3}\right)_{11}=E V_{1} Q U_{1} F=0$. This is equivalent to $V_{1} Q U_{1}=0$ since $E$ (respectively, $F$ ) has full column (respectively, row) normal rank. We now show that it requires $Q$ to take the form given in (7). Clearly

$$
V_{1} Q\left(U_{1}, U_{2}\right)=\left(0_{r_{2} \times r_{3}}, Q_{1}\right)
$$

for some $Q_{1} \in \mathbb{R} \mathbb{H}_{\infty}^{r_{2} \times\left(p_{3}-r_{3}\right)}$. This gives

$$
V_{1} Q=\left(0, Q_{1}\right) U^{-1}=Q_{1} \widetilde{U}_{2} .
$$

Next we see that

$$
\left(\begin{array}{c}
V_{1} \\
V_{2}
\end{array}\right) Q=\left(\begin{array}{c}
Q_{1} \widetilde{U}_{2} \\
Q_{2}
\end{array}\right)
$$

for some $Q_{2} \in \mathbb{R} \mathbb{H}_{\infty}^{\left(m_{3}-r_{2}\right) \times p_{3}}$, which establishes (7).

Conversely, if (7) holds for some $Q_{1} \in \mathbb{R} \mathbb{H}_{\infty}^{r_{2} \times\left(p_{3}-r_{3}\right)}$ and $Q_{2} \in \mathbb{R} \mathbb{H}_{\infty}^{\left(m_{3}-r_{2}\right) \times p_{3}}$, then so does (71), from which follows $V_{1} Q=\left(0, Q_{1}\right) U^{-1}$ which again implies $V_{1} Q U_{1}=0$.

\section{Proof of Lemma 2:}

1) $(\Leftarrow)$ In this case $B=\left\{Q_{1}^{\dagger} \widetilde{U}_{2}^{\dagger}\right\}=\left\{\left(Q_{1}^{\dagger} W\right) \widetilde{U}_{2}\right\}$, which means any element of $B$ is also an element of $A$. $\Leftrightarrow$ Suppose $B \subseteq A$, then for any $Q_{1}^{\dagger} \in \mathbb{R H}_{\infty}^{r \times c}$ there exist some $Q_{1} \in \mathbb{R} \mathbb{R}_{\infty}^{r \times c}$ such that $Q_{1}^{\dagger} \widetilde{U}_{2}^{\dagger}=Q_{1} \widetilde{U}_{2}$. Let us now choose the first row of $Q_{1}^{\dagger}$ to be $[0, \ldots, 1, \ldots, 0]$ (with the one in the $i$ th place), and all other rows to be zero, and define $q_{i}$ to be the first row of the corresponding $Q_{1}$. Then $[0, \ldots, 1, \ldots, 0] \widetilde{U}_{2}^{\dagger}=q_{i} \widetilde{U}_{2}$, from which we conclude that $\widetilde{U}_{2}^{\dagger}=W \widetilde{U}_{2}$ where $W=\left[q_{1}^{\prime}, q_{2}^{\prime}, \ldots, q_{c}^{\prime}\right]^{\prime}$.

2) From 1) we know that $B=A$ if and only if $\widetilde{U}_{2}^{\dagger}=W_{1} \widetilde{U}_{2}$ and $\widetilde{U}_{2}=W_{2} \widetilde{U}_{2}^{\dagger}$ for some $W_{1}, W_{2} \in \mathbb{R} \mathbb{M}_{\infty}^{c \times c}$. Hence we have $\widetilde{U}_{2}^{\dagger}=W_{2} W_{1} \widetilde{U}_{2}^{\dagger}$ which gives $\left(I-W_{2} W_{1}\right) \widetilde{U}_{2}^{\dagger}=$ 0 . Since $\widetilde{U}_{2}^{\dagger}$ is full row normal rank, it is equivalent to $W_{2} W_{1}=I$, which is equivalent to $W_{1}$ and $W_{2}$ being unimodular and inverses of each other over $\mathbb{R} \mathbb{R} \mathbb{H}_{\infty}$.

\section{Proof of Theorem 2:}

1) 2) These conditions allow us to choose $M=I, \tilde{M}=I$, $N=\tilde{N}=P_{22}, X=I, \tilde{X}=I, Y=0, \tilde{Y}=0$ and $V_{1}=I$. Then (8) follows directly from (3) and (7).

3) Consider $\widetilde{U}_{2}$ and $\widetilde{U}_{2}^{\dagger}$ such that all stabilizing controllers can be parameterized as $K=$ $\left(I-Q_{1} \widetilde{U}_{2} P_{22}\right)^{-1} Q_{1} \widetilde{U}_{2}, Q_{1} \in \mathbb{R} \mathbb{H}_{\infty}^{r_{2} \times\left(p_{3}-r_{3}\right)}$ and $K^{\dagger}=\left(I-Q_{1}^{\dagger} \widetilde{U}_{2}^{\dagger} P_{22}\right)^{-1} Q_{1}^{\dagger} \widetilde{U}_{2}^{\dagger}, Q_{1}^{\dagger} \in$ $\mathbb{R} \mathbb{H}_{\infty}^{r_{2} \times\left(p_{3}-r_{3}\right)}$, respectively. We can check that $K=K^{\dagger}$ if and only if $Q_{1} \widetilde{U}_{2}=Q_{1}^{\dagger} \widetilde{U}_{2}^{\dagger}$. From Lemma 2 , this means there exits a unimodular matrix $W$ such that $\widetilde{U}_{2}^{\dagger}=W \widetilde{U}_{2}$.

4) Since $U_{2}, P_{22}$ have elements in $\mathbb{R} \mathbb{H}_{\infty}$, it follows from [30, Corollary 5.5] that $K_{1}$ stabilizes $\widetilde{U}_{2} P_{22}$ if and only if $\left(I-K_{1} \widetilde{U}_{2} P_{22}\right)^{-1} K_{1} \in \mathbb{R} \mathbb{H}_{\infty}^{m_{3} \times\left(p_{3}-r_{3}\right)}$, which is equivalent to $\left(I-K_{1} \widetilde{U}_{2} P_{22}\right)^{-1} K_{1} \widetilde{U}_{2} \in \mathbb{R} \mathbb{H}_{\infty}^{m_{3}} \times p_{3}$ since $\widetilde{U}_{2}$ is right invertible over $\mathbb{R} R \mathbb{H}_{\infty}$, which is the necessary and sufficient condition that $K_{1} \widetilde{U}_{2}$ stabilizes $P_{22}$. To complete the proof, let $Q_{1}=-(I-$ $\left.K_{1} \widetilde{U}_{2} P_{22}\right)^{-1} K_{1} \in \mathbb{R} \mathbb{M} \mathbb{H}_{\infty}^{m_{3} \times\left(p_{3}-r_{3}\right)}$ and note that $K_{1}=$ $-\left(I-Q_{1} \widetilde{U}_{2} P_{22}\right)^{-1} Q_{1}$. Therefore $K=K_{1} \widetilde{U}_{2}$ will take the form of (8), from which the result follows.

5) Any stabilizing controller $K$ for which $T_{\hat{w}_{1} \rightarrow \hat{z}_{1}}=$ $\left(T_{1}\right)_{1,1}$ takes the form of (8). We can then define a controller $K_{1}=-\left(I-Q_{1} \widetilde{U}_{2} P_{22}\right)^{-1} Q_{1}$ for some $Q_{1} \in \mathbb{R} \mathbb{H}_{\infty}^{m_{3} \times\left(p_{3}-r_{3}\right)}$ such that $K=K_{1} \widetilde{U}_{2}$. It can also be shown directly that $-\left(I-K_{1} \widetilde{U}_{2} P_{22}\right)^{-1} K_{1}=$ $Q_{1} \in \mathbb{R} \mathbb{H}_{\infty}^{m_{3} \times\left(p_{3}-r_{3}\right)}$, which means that $K_{1}$ stabilizes $\widetilde{U}_{2} P_{22}$.

Proof of Corollary 1: Using the parameterization of Theorem 2(2), it follows from (4) that the closed-loop transfer function $(T)_{\hat{w}_{1} \rightarrow \hat{z}_{1}}$ remains the same as when $K_{0}=-\left(I-\bar{Q} P_{22}\right)^{-1} \bar{Q}$ is applied if and only if $\left(T_{1}+T_{2} Q T_{3}\right)_{1,1}=\left(T_{1}+T_{2} \bar{Q} T_{3}\right)_{1,1}$, which is equivalent to $\left(T_{2}(Q-\bar{Q}) T_{3}\right)_{1,1}=0$, which results in (9) by using Theorem 1 where $V_{2}, Q_{2}$ are empty. 


\section{REFERENCES}

[1] E. Bakker, L. Nyborg, and H. B. Pacejka, "Tyre modeling for use in vehicle dynamics studies," Soc. Automotive Eng. Trans., vol. 96, pp. 190-204, 1987.

[2] D. A. Crolla and A. M. A. Aboul Nour, "Theoretical comparisons of various active suspension systems in terms of performance and power requirements," in Proc. IMecE Conf. Advanced Suspensions, London, U.K., Oct. 24-25, 1988, paper C420/88, pp. 1-9.

[3] J. C. Dixon, Tyres, Suspension and Handling, 1st ed. Cambridge, U.K.: Cambridge Univ. Press, 1991.

[4] R. J. Dorling, "Integrated Control of Road Vehicle Dynamics," Ph.D., Cambridge Univ., Cambridge, U.K., 1996.

[5] B. A. Francis, "A course in $H_{\infty}$ control theory," in Lecture Notes in Control and Information Sciences. Berlin, Germany: Springer-Verlag, 1987.

[6] K. Hayakawa, K. Matsumoto, M. Yamashita, Y. Suzuki, K. Fujimori, and H. Kimura, "Robust $H_{\infty}$ feedback control of decoupled automobile active suspension systems," IEEE Trans. Automat. Contr., vol. 44, pp. 392-396, Feb. 1999.

[7] J. K. Hedrick and T. Butsuen, "Invariant properties of automotive suspensions," Proc. Inst. Mech. Eng. J. Automobile Eng., pt. D, vol. 204 pp. 21-27, 1990.

[8] D. Hrovat and M. Hubbard, "Optimal vehicle suspensions minimizing RMS rattlespace, sprung-mass acceleration and jerk," Trans. Amer. Soc. Mech. Eng., vol. 103, pp. 228-236, 1981.

[9] D. Hrovat, "A class of active LQG optimal actuators," Automatica, vol. 18, pp. 117-119, 1982

[10] D. Karnopp, "Theoretical limitations in active suspension," Veh. Syst. Dyn., vol. 15, pp. 41-54, 1986.

[11] D. C. McFarlane and K. Glover, "Robust control design using normalized coprime factor plant descriptions," in Lecture Notes in Control and Information Sciences. Berlin, Germany: Springer-Verlag, 1987.

[12] K. M. Malek and J. K. Hedrick, "Decoupled active suspension design for improved automotive ride quality/handling performance," Veh. Syst. Dyn., vol. 15, supplement, pp. 383-398, 1986.

[13] P. E. Mercier, "Vehicle suspensions-A theory and analysis that accord with experiment," Automobile Eng., vol. 32, pp. 405-410, 1942.

[14] K. Park and J. J. Bongiorno Jr, "Wiener-Hopf design of servo-regulator-type multivariable control systems including feedforward compensation," Int. J. Contr., vol. 52, no. 5, pp. 1189-1216, 1990.

[15] R. Pitcher, H. Hillel, and C. M. Curtis, "Hydraulic suspensions with particular reference to public service vehicles," in Proc. Public Service Veh. Conf., 1977.

[16] R. S. Sharp and S. A. Hassan, "On the performance capabilities of active automobile suspension systems of limited bandwidth," Veh. Syst. Dyn., vol. 16 , pp. 213-225, 1987 .

[17] _ - "The relative performance capabilities of passive, active and semiactive car suspension systems," Proc. Inst. Mech. Eng., vol. 200, pp. 219-228, 1986

[18] M. C. Smith, "Achievable dynamic response for automotive active suspension," Veh. Syst. Dyn., vol. 24, pp. 1-33, 1995.

[19] M. C. Smith and G. W. Walker, "Performance limitations and constraints for active and passive suspension: A mechanical multi-port approach," Veh. Syst. Dyn., vol. 33, pp. 137-168, 2000

[20] A. G. Thompson, "Design of active suspensions," Proc. Inst. Mech. Eng., vol. 185, pp. 553-563, 1970-71.

[21] M. Vidyasagar, Control System Synthesis: A Factorization Approach. Cambridge, MA: MIT Press, 1985.

[22] G. W. Walker, "Constraints Upon the Achievable Performance of Vehicle Suspension Systems," Ph.D. dissertation, Cambridge Univ., Cambridge, U.K., 1997.
[23] F.-C. Wang and M. C. Smith, "Active and passive suspension control for vehicle dive and squat," in Automotive Control Workshop, Lund, Sweden, May 18-19, 2001. To appear in "Nonlinear and Hybrid Systems in Automotove Control," R. Johansson and A Rantzer, Eds. London, U.K.: Springer-Verlag.

[24] R. A. Williams and A. Best, "Control of a low frequency active suspension," Control' '94, vol. 1, no. 389, pp. 338-343.

[25] R. A. Williams, A. Best, and I. L. Crawford, "Refined low frequency active suspension," in Proc. Int. Conf. Vehicle Ride Handling, Birmingham, U.K., Nov. 1993, C466/028, pp. 285-300.

[26] P. G. Wright and D. A. Williams, "The application of active suspension to high performance road vehicles," in Proc. IMecE Conf. Microproces sors Fluid Power Eng., London, U.K., 1984, paper C239/84, pp. 23-28.

[27] — " "The case for an irreversible active suspension system," SAE Trans., J. Passenger Cars, pp. 83-90, 1989.

[28] D. C. Youla, H. A. Jabr, and J. J. Bongiorno Jr, "Modern Wiener-Hopf design of optimal controllers-Part II: The multivariable case," IEEE Trans. Automat. Contr., vol. AC-21, pp. 319-338, 1976.

[29] D. C. Youla and J. J. Bongiorno Jr, "A feedback theory of two-degree-offreedom optimal Wiener-Hopf design," IEEE Trans. Automat. Contr., vol. AC-30, pp. 652-665, 1985.

[30] K. Zhou, J. C. Doyle, and K. Glover, Robust and Optimal Control. Upper Saddle River, NJ: Prentice-Hall, 1996.

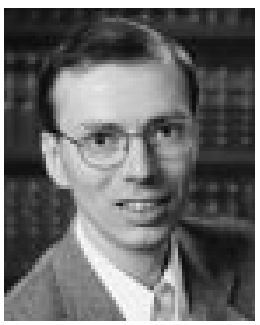

Malcolm C. Smith (M'90-SM'00-F'02) received the B.A. degree in mathematics in 1978, the M.Phil. degree in control engineering and operational research in 1979, and the Ph.D. degree in control engineering in 1982 from Cambridge University, Cambridge, U.K.

He was a Research Fellow at the German Aerospace Center, Oberpfaffenhofen, Germany, a Visiting Assistant Professor and Research Fellow with the Department of Electrical Engineering, McGill University, Montreal, QC, Canada, and an Assistant Professor with the Department of Electrical Engineering, The Ohio State University, Columbus. In 1990, he joined the Engineering Department at the University of Cambridge, U.K., as a Lecturer and subsequently Reader in 1997. His research interests include robust control, nonlinear systems, and automotive applications.

Dr. Smith is a co-recipient with Dr. Tryphon T. Georgiou of the 1992 and 1999 George Axelby Best Paper Awards in the IEEE TRANSACTIONS ON AUTOMATIC CONTROL.

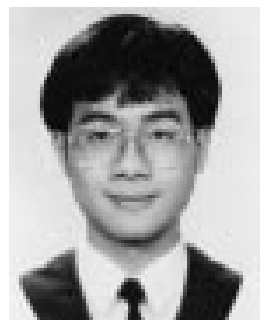

Fu-Cheng Wang ( $\left.\mathrm{S}^{\prime} 00\right)$ was born in Taipei, Taiwan, R.O.C., in 1968. He received the B.S. and M.S. degrees in mechanical engineering from National Taiwan University, Taipei, Taiwan, R.O.C., in 1990 and 1992, respectively, and the Ph.D. degree in control engineering from Cambridge University, Cambridge, U.K., in 2002.

Since 2002, he has been a Research Associate in the Control Group of the Engineering Department, Cambridge University. His research interests include $H_{\infty}$ control, vehicle active/passive suspension control, loop-shaping, and fuzzy systems. 